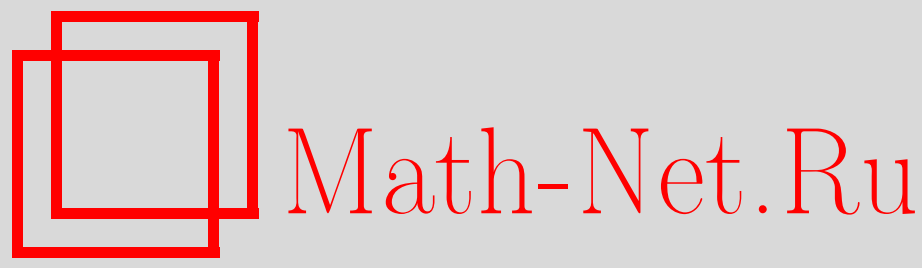

В. А. Топчий, Теоремы двумерного восстановления при слабых моментных ограничениях и критические ветвящиеся процессы Беллмана-Харриса, Дискрет. матем., 2015, том 27, выпуск 1, 123-145

DOI: https://doi.org/10.4213/dm1320

Использование Общероссийского математического портала Math-Net.Ru подразумевает, что вы прочитали и согласны с пользовательским соглашением http://www.mathnet.ru/rus/agreement

Параметры загрузки:

IP : 34.229 .45 .116

26 апреля 2023 г., 14:15:20

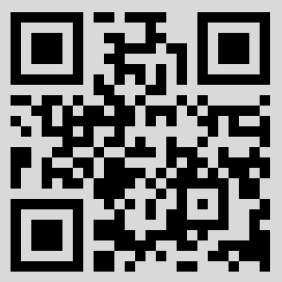




\title{
Теоремы двумерного восстановления при слабых моментных ограничениях и критические ветвящиеся процессы Беллмана-Харриса
}

\author{
() 2015 г. В. А. Топчий*
}

Критические процессы Беллмана-Харриса с двумя типами частиц, у которых хвосты распределения продолжительности жизни имеют порядок $o\left(t^{-2}\right)$ для первого типа частиц и правильно меняются с индексом от -1 до 0 для второго, связаны с матрицами двумерного восстановления специального вида. Ранее в публикациях В.А. Ватутина и В.А. Топчия асимптотика этих матриц и их приращений первого и второго порядков позволила доказать ряд предельных теорем для исходных ветвящихся процессов. Мы описываем свойства таких матриц восстановления при существенно ослабленных условиях на распределения продолжительности жизни частиц, а затем применяем результаты к описанию асимптотики ряда моментов и их приращений для ветвящихся процессов.

Работа выполнена при финансовой поддержке РФФИ, проект № 14-01-00318.

Ключевые слова: матрицы двумерного восстановления, приращения матриц, критические процессы Беллмана-Харриса с двумя типами частиц, частицы с бесконечным средним времени жизни, асимптотические формулы, правильно меняющиеся функции.

\section{1. Введение, основные определения}

В данной работе теоремы о свойствах матриц восстановления из [1] распространены на более широкий класс распределений. Работа [2] была одной из первых, где исследовались многомерные критические процессы Беллмана-Харриса с правильно меняющимися хвостами продолжительности жизни частиц с индексом, принадлежащим полуинтервалу $[-1,0)$. Для изучения таких процессов с одним типом частиц необходимы теоремы восстановления, доказанные в [3], и их усиления из [4]. В многомерном случае соответствующие теоремы восстановления доказаны в [2]. Если скорости убывания хвостов продолжительности жизни частиц различных типов имеют различные порядки, то при описании свойств короткоживущих частиц стандартные нормировки приводят к вырожденным распределениям, а при отличающихся по порядку нормирующих функциях, как показано в [1], теоремы о свойствах приращений первого и второго порядков для функций восстановления позволяют получить ряд

* Место работы: Институт математики им. С. Л. Соболева СО РАН, e-mail: topchij@ofim.oscsbras.ru 
новых асимптотических результатов. Упомянутые теоремы о свойствах матриц восстановления представляют самостоятельный теоретический интерес и важны для различных приложений. В дальнейшем мы часто будем ссылаться на утверждения и формулы из работы [1]. Для краткости мы будем приписывать им индекс [1]. Например, (1.6) ${ }_{[1]}$ обозначает, что это формула (1.6) из [1].

Начнем с формулировки основных понятий и соотношений из [1].

Матрицы восстановления определим с помощью ветвящихся процессов Беллмана-Харриса $\mathbf{Z}(t)=\left(Z_{1}(t), Z_{2}(t)\right)$ с двумя типами частиц. Частицы $i$-го типа $(i=1,2)$ с распределением продолжительности жизни $G_{i}(t)$ в момент гибели порождают $\xi_{i 1}$ потомков первого типа и $\xi_{i 2}$ второго с распределениями, задаваемыми производящими функциями

$$
f_{i}(\mathbf{s})=f_{i}\left(s_{1}, s_{2}\right):=\mathbf{E} s_{1}^{\xi_{i 1}} s_{2}^{\xi_{i 2}}, \mathbf{s}:=\left(s_{1}, s_{2}\right) \in[0,1]^{2} .
$$

Используя символ транспонирования $T$ для двумерных векторов в вектор-столбцы, положим $\mathbf{G}(t):=\left(G_{1}(t), G_{2}(t)\right)^{T}, \mathbf{f}(\mathbf{s}):=\left(f_{1}(\mathbf{s}), f_{2}(\mathbf{s})\right)^{T}$. Символ $\mathbf{1}$ используется для обозначения векторов $(1,1)$ и $(1,1)^{T}$, а символ $\mathbf{0}$ - для обозначения векторов $(0,0),(0,0)^{T}$ и нулевых матриц (в зависимости от контекста).

Пусть

$$
\begin{aligned}
m_{i j} & :=\mathbf{E} \xi_{i j}=\left.\frac{\partial f_{i}(\mathbf{s})}{\partial s_{j}}\right|_{\mathbf{s}=\mathbf{1}}, b_{j k}^{i}:=\mathbf{E} \xi_{i j} \xi_{i k}=\left.\frac{\partial^{2} f_{i}(\mathbf{s})}{\partial s_{j} \partial s_{k}}\right|_{\mathbf{s}=\mathbf{1}}, i, j, k=1,2, \\
\mathbf{M}(t) & :=\left(\begin{array}{ll}
m_{11} G_{1}(t) & m_{12} G_{1}(t) \\
m_{21} G_{2}(t) & m_{22} G_{2}(t)
\end{array}\right), \mathbf{M}=\mathbf{M}(\infty):=\left(\begin{array}{ll}
m_{11} & m_{12} \\
m_{21} & m_{22}
\end{array}\right) .
\end{aligned}
$$

Условия неразложимости и непериодичности для критического процесса Беллмана-Харриса $\mathbf{Z}(t)$ в терминах матрицы $\mathbf{M}$ означают, что верны неравенства $\mathbf{M}^{2}>\mathbf{0}, m_{i i}<1, m_{11}+m_{22}>0$ и $m_{i j}>0$ при $i \neq j$, перронов корень матрицы М равен 1, и, следовательно, найдутся такие левый и правый собственные векторы $\mathbf{v}=\left(v_{1}, v_{2}\right)$ и $\mathbf{u}=\left(u_{1}, u_{2}\right)$, что

$$
\mathbf{M} \mathbf{u}^{T}=\mathbf{u}^{T}, \mathbf{v M}=\mathbf{v}, \mathbf{v} \mathbf{u}^{T}=1, \mathbf{u}>\mathbf{0}, \mathbf{v}>\mathbf{0}, \mathbf{v} \mathbf{1}=1 .
$$

Пусть вторые моменты численностей потомства у обоих типов частиц конечны и

$$
B:=\frac{1}{2} \sum_{i, j, k=1}^{2} v_{i} b_{j k}^{i} u_{j} u_{k}<\infty .
$$

Положим $\mu_{i}(t):=\int_{0}^{t}\left(1-G_{i}(w)\right) d w$ с $\mu_{i}:=\mu_{i}(\infty) \leqslant \infty$ для $i=1,2$. Обозначим через $\mathcal{L}$ класс медленно меняющихся на бесконечности функций, определенных на $\mathbb{R}^{+}$. Далее предполагаем, что при $t \rightarrow \infty$

$$
\begin{gathered}
1-G_{1}(t)=o\left(t^{-2}\right), \\
1-G_{2}(t)=\ell(t) t^{-\beta}, \beta \in(0,1] \text { и } \mu_{2}=\infty, \text { если } \beta=1,
\end{gathered}
$$

где $\ell(t) \in \mathcal{L}$. Заметим, что условия (4) и (5) влекут $\mu_{1}<\infty$ и $\mu_{2}=\infty$ для всех $\beta \in(0,1]$.

В асимптотических соотношениях типа $a(t)=O(b(t))$ или $a(t)=o(b(t)), a(t) \sim b(t)$ или $\lim a(t)=a$ считаем, что $t \rightarrow \infty$. Запись $a(t) \asymp b(t)$ означает, что $a(t)=O(b(t))$ и $b(t)=O(a(t))$. 
Так как $\mu_{2}=\infty$ при $\beta=1$, то условие (5) влечет

$$
\mu_{2}(t) \sim\left\{\begin{array}{lll}
t^{1-\beta} \ell(t)(1-\beta)^{-1}, & \text { если } & \beta \in[0,1), \\
\ell_{1}(t), & \text { если } & \beta=1,
\end{array}\right.
$$

где $\ell_{1}(t):=\int_{0}^{t} \ell(u) u^{-1} d u \rightarrow \infty$ при $t \rightarrow \infty$, причем $\ell_{1}(t) \in \mathcal{L}$ и $\ell(t)=o\left(\ell_{1}(t)\right)$ (см., например, лемму и теорему 1 из [5; гл.VIII, §9], соответственно). Следовательно, при выполнении условия (5)

$$
R(t):=\frac{t}{\mu_{2}(t)} \sim \begin{cases}(1-\beta) t^{\beta} \ell^{-1}(t), & \text { если } \beta \in(0,1), \\ t \ell_{1}^{-1}(t), & \text { если } \beta=1 .\end{cases}
$$

В работе [1] на свойства функций $G_{i}(t), i=1,2$, накладывались ограничения двух основных типов, а именно, Гипотезы $\mathbf{A}$ и В. Сформулируем первую из них.

Гипотеза А. Функиия распределения $G_{1}(t)$ удовлетворяет условию (4), распределение $G_{2}(t)$ - условию (5) при $\beta \in(0,1]$, причем при $\beta \in(0,1 / 2]$ дополнительно предполагается, что существуют такие постоянные $C$ u $T_{0}$, что при $t \geqslant T_{0} u$ любом фиксированном $\Delta>0$

$$
G_{2}(t+\Delta)-G_{2}(t) \leqslant C \Delta \ell(t) t^{-\beta-1}
$$

Гипотеза В из работы [1] включает в себя, в частности, излишне ограничительное условие $1-G_{1}(t)=o\left(t^{-2-\beta-\delta}\right)$ для некоторого $\delta>0$ и будет заменяться более слабыми Гипотезами $\mathbf{A}^{+}$или $\mathbf{A}^{++}$в зависимости от требуемой точности оценок. Отметим, что последние более ограничительны, чем Гипотеза $\mathbf{A}$.

Гипотеза $\mathbf{A}^{+}$. Функция распределения $G_{1}(t)$ удовлетворяет условию (4), а распределение $G_{2}(t)$ - условию (5) и имеет такую абсолютно непрерывную плотность $g_{2}(t)$, что для $\beta \in(0,0.5]$ и некоторого $C>0$

$$
g_{2}(t) \leqslant C \ell(t)(t+1)^{-\beta-1},
$$

$a$, в случае $\beta \in(0.5,1]$ плотность $g_{2}(t)$ абсолютно непрерывна, $\left|g_{2}^{\prime}(t)\right|$ интегрируе$\mathcal{M} a$,

$$
g_{2}(t) \sim \beta t^{-\beta-1} \ell(t),
$$

и для некоторой постоянной $C \geqslant 1$

$$
\underset{u>t}{\operatorname{Var}} u g_{2}(u) \sim C \beta t^{-\beta} \ell(t)
$$

В случае $\beta=1$ условие (4) заменяется следующим: найдется такое $\Delta>0$, что

$$
G_{1}(t+\Delta)-G_{1}(t)=o\left(t^{-2} \min \{1, \ell(t)\}\right) .
$$

Очевидно, что (8) влечет (7) (как и (9) влечет (7)), а условия Гипотезы $\mathbf{A}^{+}$влекут справедливость Гипотезы А. Отметим, что если (11) верно для некоторого $\Delta>0$, то оно верно и при всех $\Delta>0$. Это относится и к условию (18), приводимому ниже.

Введем условия, ограничивающие приращения $G_{1}(t)$ на сравнительно небольших (возможно, растущих одновременно с параметром $t$ ) интервалах, вместо жестких аналогов для хвостов $1-G_{1}(t)$. 
Далее мы будем использовать хорошо известный факт (см., например, формулу (9.9) в [5; гл.VIII, §9]): если функция распределения $G_{2}(t)$ удовлетворяет условию (5), то существует такое распределение $G_{2}^{c}(t)$, что

$$
1-G_{2}(t) \sim 1-G_{2}^{c}(t)=t^{-\beta} \ell^{c}(t),
$$

где $\ell^{c}(t) \sim \ell(t)$ дифференцируемая медленно меняющаяся функция и $\left(\ell^{c}(t)\right)^{\prime}=$ $o\left(\ell^{c}(t) t^{-1}\right)$.

Пусть $\gamma \in(0,1]$ - произвольное фиксированное число,

$$
\mathcal{N}_{\gamma}(t)=\sup \left\{\tau: \tau^{-(\beta+2) \gamma^{-1}} \ell^{c}\left(\tau^{\gamma^{-1}}\right)=1-G_{2}^{c}(t)\right\}
$$

и $T_{0}=\sup \left\{t: t / 2-2 \mathcal{N}_{\gamma}(t) \leqslant 1, g_{2}(t)>0\right\}$. Выбор $T_{0}$ обеспечивает корректность соотношений при доказательстве леммы 3 и дифференцируемость $\mathcal{N}_{\gamma}(t)$ при $t>T_{0}$. Очевидно, что функция $\mathcal{N}_{\gamma}(t)$ монотонна. Более того, так как

$$
\mathcal{N}_{\gamma}^{-(\beta+2) \gamma^{-1}}(t) \ell\left(\mathcal{N}_{\gamma}^{\gamma^{-1}}(t)\right) \sim t^{-\beta} \ell(t),
$$

то с помощью теоремы 1.5 .12 из [6; c.28] легко получить, что $\mathcal{N}_{\gamma}(t)$ правильно меняется с параметром $\beta \gamma /(\beta+2)$ на бесконечности. Это и дифференцируемость функции $\ell^{c}(t)$ позволяет записать соотношения

$$
\mathcal{N}_{\gamma}(t)=t^{\beta \gamma(\beta+2)^{-1}} \ell_{\mathcal{N}_{\gamma}}(t), \frac{\mathcal{N}_{\gamma}^{\prime}(t)}{\mathcal{N}_{\gamma}(t)} \sim \frac{\beta \gamma}{\beta+2} t^{-1}, \frac{\mathcal{N}_{\gamma}(x t)}{\mathcal{N}_{\gamma}(t)} \sim x^{\beta \gamma(\beta+2)^{-1}},
$$

где $l_{\mathcal{N}_{\gamma}}(t) \in \mathcal{L}$ и $x>0$ - любое фиксированное число.

Гипотеза $\mathbf{A}^{++}$. Функиия распределения $G_{1}(t)$ удовлетворяет условию (4), а распределение $G_{2}(t)$ - условию (5) и имеет абсолютно непрерывную плотность $g_{2}(t)$. Кроме того:

i) если $\beta \in(0,0.5]$, то существуют такие постоянные $c^{*}>0, T>0$ и $\gamma \in(0,1]$, uто при $t \geqslant T$ и $y \in\left(1,10 t^{\beta \gamma}\right]$

$$
G_{1}(t)-G_{1}\left(t-y \mathcal{N}_{\gamma}(t)\right) \leqslant c^{*} y t^{-\beta-2} \ell(t)
$$

и справедлива оченка

$$
g_{2}^{\prime}(t) \leqslant-C(t+1)^{-\beta-2} \ell(t)
$$

ii) если $\beta \in(0.5,1)$, то существует такая постоянная $\gamma \in(0, \min \{1,(1-\beta)(\beta+$ $2) / \beta\}]$, что при $y \in\left(1,10 t^{\beta \gamma}\right]$

$$
G_{1}(t)-G_{1}\left(t-y \mathcal{N}_{\gamma}(t)\right)=o\left(y t^{-\beta-2} \ell(t)\right),
$$

$g_{2}^{\prime}(t)$ абсолютно непрерывна, $\left|g_{2}^{\prime \prime}(t)\right|$ интегрируема,

$$
g_{2}^{\prime}(t) \sim-(\beta+1) \beta t^{-\beta-2} \ell(t)
$$

и для некоторого $C \geqslant 1$

$$
\operatorname{Var}_{u>t} u^{2} g_{2}^{\prime}(u) \sim C(\beta+1) \beta t^{-\beta} \ell(t) ;
$$

iii) если $\beta=1$, то найдется такое $\Delta>0$, что

$$
G_{1}(t+\Delta)-G_{1}(t)=o\left(t^{-3} \min \{1, \ell(t)\}\right),
$$

$\left|\ell^{\prime}(t)\right| t \in \mathcal{L}$ и для некоторого $C_{1} \geqslant 1$

$$
\operatorname{Var}_{u>t}\left|l^{\prime}(u)\right| \sim C_{1}\left|\ell^{\prime}(t)\right| .
$$

Очевидно, что из Гипотезы $\mathbf{A}^{++}$следуют условия Гипотезы $\mathbf{A}^{+}$. Напомним, что согласно $(6) \mu_{2}(t) \sim \ell_{1}(t) \in \mathcal{L}$, если $\beta=1$. 


\section{2. Свойства матриц восстановления}

Напомним ряд определений и утверждений из раздела $2_{[1]}$, а потом докажем более точные утверждения при справедливости Гипотез $\mathbf{A}^{+}$или $\mathbf{A}^{++}$.

Пусть $\mathbf{I}(t):=1_{\{t \geqslant 0\}} \mathbf{I}$ - произведение индикатора $1_{\{t \geqslant 0\}}$ на матрицу $\mathbf{I}:=\left(\delta_{i j}\right)_{i, j=1}^{2}$, где $\delta_{i j}$ - символ Кронекера. Для матриц $\mathbf{A}(t)=\left(A_{i j}(t)\right)_{i, j=1}^{2}$ и $\mathbf{B}(t)=\left(B_{i j}(t)\right)_{i, j=1}^{2}$ определим свертку $\mathbf{C}(t)=\mathbf{A} * \mathbf{B}(t)=\left(C_{i j}(t)\right)_{i, j=1}^{2}$ с помощью соотношений $C_{i j}(t):=$ $\sum_{k=1}^{2} A_{i k} * B_{k j}(t)$. Аналогично определяется свертка матрицы и вектора.

Обозначим $\mathbf{M}^{* 0}(t):=\mathbf{I}(t)$ и введем матрицу восстановления

$$
\mathbf{U}(t)=\left(U_{i j}(t)\right)_{i, j=1}^{2}:=\sum_{k=0}^{\infty} \mathbf{M}^{* k}(t) .
$$

При этом считаем, что при $t<0$ все элементы матрицы $\mathbf{U}(t)$ равны нулю. В данной работе это же предполагается и для всех матричных и одномерных функций. Очевидно, что

$$
\mathbf{U}(t)=\mathbf{I}(t)+\mathbf{M} * \mathbf{U}(t) .
$$

Пусть $\Gamma(a)$ - стандартная функция Эйлера (см. $\left[5 ;\right.$ гл.II, §2]) и $B_{a, b}=$ $=\Gamma(a+b)(\Gamma(a) \Gamma(b))^{-1}$. Положим $\Gamma_{1} \equiv 1$, а если $\beta \in(0,1)$, то

$$
\Gamma_{\beta}:=(\beta \Gamma(\beta) \Gamma(2-\beta))^{-1}=B_{\beta, 1-\beta}(1-\beta)^{-1} \beta^{-1}=(1-\beta)^{-1} \beta^{-1} \pi^{-1} \sin \pi \beta .
$$

Введем матрицу $\mathbf{D}=\left(D_{i j}\right)_{i, j=1}^{2}$ с положительными элементами

$$
D_{i i}=\left(1-m_{j j}\right)\left(1-m_{11}\right)^{-1} \text { и } D_{i j}=m_{i j}\left(1-m_{11}\right)^{-1}, i \neq j .
$$

Для распределения $G(t)$ неотрицательной случайной величины и постоянной $a \in$ $(0,1]$ положим $\mu_{G}(t):=\int_{0}^{t}(1-G(w)) d w$ и

$$
U_{a G}(t):=\sum_{k=0}^{\infty} a^{k} G^{* k}(t), t \geqslant 0 .
$$

Тогда $U_{G}(t):=U_{1 G}(t)$ будет функцией восстановления для $G(t)$. Плотности для мер $U_{a G}(t)$ и функций восстановления $U_{G}(t)$, если они абсолютно непрерывны, будем обозначать $u_{a G}(t)$ и $u_{G}(t)$, соответственно.

В [1] введены функции распределения (см. $\left.(2.18)_{[1]},(2.20)_{[1]}\right)$

$$
\begin{aligned}
& H_{j}(t):=\left(1-m_{j j}\right) \sum_{s=0}^{\infty} m_{j j}^{s} G_{j}^{* s}(t)=\left(1-m_{j j}\right) U_{m_{j j} G_{j}}(t), \\
& X_{j}(t)=m_{i i} G_{i}(t)+\left(1-m_{i i}\right) G_{i} * G_{j} * H_{j}(t), \quad i \neq j .
\end{aligned}
$$

Определим матрицы

$$
\mathbf{U}^{d}(t)=\left(U_{i j}^{d}(t)\right)_{i, j=1}^{2}:=\left(\begin{array}{cc}
\frac{H_{1}(t)}{1-m_{11}} & \frac{m_{12} G_{1} * H_{1}(t)}{1-m_{11}} \\
0 & 0
\end{array}\right)
$$

и $\mathbf{U}^{c}(t):=\mathbf{U}(t)-\mathbf{U}^{d}(t)$. 
Теорема 1. Пусть верно условие (4), тогда для $i=1,2$

$$
\left(\delta_{i 1}+\delta_{i 2} m_{12}\right)\left(1-m_{11}\right)^{-1}-U_{1 i}^{d}(t)=o\left(t^{-2}\right) \quad u \quad U_{2 i}^{d}(t) \equiv 0 .
$$

В частности, $U_{1 i}^{d}(t+\Delta)-U_{1 i}^{d}(t)=o\left(t^{-2}\right)$ при любом $\Delta>0$.

Если $\beta=1$ и верно (11), то

$$
U_{1 i}^{d}(t+\Delta)-U_{1 i}^{d}(t)=o\left(t^{-2} \min \{1, \ell(t)\}\right) .
$$

Теорема 2. Пусть верна Гипотеза $\boldsymbol{A}^{+}$. Тогда матрица $\mathbf{U}^{c}(t)$ абсолютно непрерывна, а ее плотность $\mathbf{u}^{c}(t)$ имеет асимптотику

$$
\mathbf{u}^{c}(t) \sim \beta \Gamma_{\beta} \mu_{2}^{-1}(t) \mathbf{D} .
$$

Для описания асимптотики $\left(\mathbf{u}^{c}(t)\right)^{\prime}$ нам необходима Гипотеза $\mathbf{A}^{++}$.

Теорема 3. Пусть верна Гипотеза $\boldsymbol{A}^{++}$. Тогда плотность $\mathbf{u}^{c}(t)$ абсолютно непрерьвна, причем при $\beta \in(0,1)$ верно соотношение

$$
\left(\mathbf{u}^{c}(t)\right)^{\prime} \sim(\beta-1) \beta \Gamma_{\beta} t^{-1} \mu_{2}^{-1}(t) \mathbf{D},
$$

а при $\beta=1$

$$
\left(\mathbf{u}^{c}(t)\right)^{\prime} \sim-\ell(t) t^{-1} \ell_{1}^{-2}(t) \mathbf{D} .
$$

Более того, для $i=1,2$

$$
U_{i 1}^{d}(t)-U_{i 1}^{d}\left(t-\mathcal{N}_{\gamma}(t)\right)=\left\{\begin{array}{lll}
O\left(t^{-\beta-2} \ell(t)\right), & \text { если } & \beta \in(0,0.5], \\
o\left(t^{-\beta-2} \ell(t)\right), & \text { если } & \beta \in(0.5,1),
\end{array}\right.
$$

а если $\beta=1$, то при любом фиксированном $\Delta>0$

$$
U_{i 1}^{d}(t+\Delta)-U_{i 1}^{d}(t)=o\left(t^{-3} \min \{1, \ell(t)\}\right) .
$$

Основываясь на [1], приведем явный вид функций, фигурирующих в приведенных выше теоремах.

Матрица восстановления $\mathbf{U}(t)\left(\right.$ см. $\left.(2.10)_{[1]},(2.21)_{[1]}\right)$ допускает представление

$$
U_{i i}(t)=U_{X_{j}}(t), U_{i j}(t)=\frac{m_{i j}}{1-m_{j j}} G_{i} * H_{j} * U_{i i}(t), i=1,2, i \neq j,
$$

a ее главную часть $\mathbf{U}^{c}(t)\left(\right.$ см. $\left.(2.30)_{[1]}-(2.32)_{[1]}\right)$ можно определить равенством

$$
U_{i j}^{c}(t):=V_{i j} * U_{X_{1}}(t), i, j=1,2,
$$

где

$$
\begin{array}{lll}
V_{11}(t):=\frac{\left(X_{1}(\cdot)-m_{22} G_{2}(\cdot)\right) * H_{1}(t)}{1-m_{11}}, & V_{12}(t):=\frac{m_{12} G_{1} * X_{1} * H_{1}(t)}{1-m_{11}}, \\
V_{21}(t):=\frac{m_{21} G_{2} * H_{1}(t)}{1-m_{11}}, & V_{22}(t):=1 .
\end{array}
$$

Отметим, что, используя последние представления, все свойства гладкости и абсолютной непрерывности для $G_{2}(t)$ легко перенести сначала на $X_{1}(t)$, затем на $U_{X_{1}}(t)$, а в силу $(30)$ и на $U_{i j}^{c}(t)$. Это отражено в утверждениях теорем 2 и 3.

Пусть $B_{\beta}=B_{\beta, 1-\beta}=\pi^{-1} \sin (\pi \beta)$, если $\beta \in(0,1), B_{1}=1$ и

$$
\mathbf{G}_{\mathbf{I}}(t):=\left(G_{i}(t) \delta_{i j}\right)_{i, j=1}^{2} .
$$

Согласно лемме 5 из [1] справедливо следующее утверждение. 
Лемма 1. Пусть выполняются условия (4) и (5). Тогда

$$
\begin{gathered}
1-H_{1}(t)=o\left(t^{-2}\right), 1-H_{2}(t) \sim m_{22} \ell(t)\left(1-m_{22}\right)^{-1} t^{-\beta}, \\
1-X_{j}(t) \sim\left(1-m_{11}\right) \ell(t)\left(1-m_{j j}\right)^{-1} t^{-\beta}, j=1,2 .
\end{gathered}
$$

Лемма 2. Пусть выполняется условие (11). Тогда при тех же $\Delta>0$

$$
H_{1}(t+\Delta)-H_{1}(t)=o\left(t^{-2} \min \{1, \ell(t)\}\right) .
$$

Доказательство. Эту лемму можно доказать при помощи рассуждений, используемых ниже при доказательстве леммы 4. Необходимые изменения будут указаны в ходе доказательства последней леммы.

Объединим теорему 8.3 из [7] для $\beta \in(0,0.5]$ и теорему 2 из [8] для $\beta \in(0.5,1]$ и запишем их в виде теоремы 4.

Теорема 4. Пусть функиия распределения $G(t)=1-t^{-\beta} \ell_{G}(t)$, где $\beta \in(0,1]$, а $l_{G}(t) \in \mathcal{L}$, абсолютно непрерывна с плотностъю $g(t)$, обладающей следующими свойствами:

i) если $\beta \in(0,0.5]$, то найдется такая постоянная $C_{0}>0$, что

$$
g(t) \leqslant C_{0}(t+1)^{-\beta-1} \ell_{G}(t)
$$

ii) если $\beta \in(0.5,1]$ и кроме того $\mu_{G}(\infty)=\infty$ в случае $\beta=1$, то плотность $g(t)$ абсолютно непрерывна, $\left|g^{\prime}(t)\right|$ интегрируема,

$$
g(t) \sim \beta t^{-\beta-1} \ell_{G}(t),
$$

u найдется такая постоянная $C \geqslant 1$, что

$$
\underset{u>t}{\operatorname{Var}} u g(u) \sim C \beta t^{-\beta} \ell_{G}(t) .
$$

Тогда для плотности $u_{G}(t)$ функции восстановления $U_{G}(t)$ верно представление

$$
u_{G}(t) \sim \beta \Gamma_{\beta} \mu_{G}^{-1}(t) .
$$

Отметим, что функция распределения $X_{1}(t)$, определенная в $(22)$, будет абсолютно непрерывной, если таковой является $G_{2}(t)$. Если верна Гипотеза $\mathbf{A}^{+}$, то плотность $x_{1}(t)$ распределения $X_{1}(t)$ имеет вид $x_{1}(t)=m_{22} g_{2}(t)+\left(1-m_{22}\right) g_{2} * G_{1} * H_{1}(t)$. Простейшие свойства сверток (см., например, лемму 5 из [1]) дают возможность убедиться в том, что при справедливости Гипотезы $\mathbf{A}^{+}$

$$
x_{1}(t)=\left\{\begin{array}{lll}
O\left(t^{-\beta-1} \ell(t)\right), & \text { если } & \beta \in(0,0.5], \\
\beta t^{-\beta-1} \ell(t)(1+o(1)), & \text { если } & \beta \in(0.5,1] .
\end{array}\right.
$$

Очевидно, что $x_{1}(t)$ абсолютно непрерывна и $\left|x_{1}^{\prime}(t)\right|$ интегрируема, если таковыми свойствами обладают $g_{2}(t)$ и $\left|g_{2}^{\prime}(t)\right|$, и для $x_{1}(t)$ верны $(35)$ и $(36)$, если для $g_{2}(t)$ верны (9) и (10), поскольку функции ограниченной вариации представимы в виде разности двух монотонных функций, то есть $t g_{2}(t)$ представима в виде разности двух монотонных функций $C_{ \pm} \beta t^{-\beta} l(t)$, где $C=C_{+}-C_{-}$.

Отсюда, из теоремы 4 и соотношения (33) при справедливости Гипотезы $\mathbf{A}^{+}$следует соотношение

$$
u_{X_{1}}(t) \sim \beta \Gamma(\beta) \mu_{2}^{-1}(t)
$$


Доказательство теоремы 1. Лемма 1 влечет первую часть теоремы 1. Вторая часть следует из (21), (23) и леммы 2.

Доказательство теоремы 2. В силу $(20) V_{i j}(\infty)=D_{i j}$. В соответствии с $(31)$ функции $V_{i j}(t)$, исключая случай $i=j=2$, когда $V_{22}(t)=1$, являются линейными комбинациями сверток абсолютно непрерывной функции $X_{1}(t)$ или (и) $G_{2}(t)$ с функциями из множества $U_{m_{11} G_{1}}(t)$ и $G_{i}(t), i=1,2$, а, следовательно, тоже абсолютно непрерывны. Пусть $v_{i j}(t)$ - плотность $V_{i j}(t)$.

Воспользуемся стандартными свойствами сверток правильно меняющихся функций и оценками для них, приведенными в лемме 5 из [1]. Докажем, что при выполнении Гипотезы $\mathbf{A}^{+}$выполняется соотношение $v_{i j}(t)=O\left(t^{-\beta-1} \ell(t)\right)$, если $\beta \in(0,0.5]$ и $v_{i j}(t) \sim \beta t^{-\beta-1} \ell(t)$, если $\beta \in(0.5,1]$. Рассмотрим свертку абсолютно непрерывного распределения $F(t)$ на $\mathbb{R}^{+}$с ограниченной непрерывной плотностью

$$
f(t)= \begin{cases}O\left(t^{-\beta-1} \ell_{F}(t)\right), & \text { если } \quad \beta \in(0,0.5], \\ \beta t^{-\beta-1} \ell_{F}(t)(1+o(1)), & \text { если } \quad \beta \in(0.5,1],\end{cases}
$$

где $\ell_{F}(t) \in \mathcal{L}$, и такого распределения $G(t)$, что $1-G(t)=o\left(t^{-2}\right)$, причем при $\beta=1$ для $G(t)$ справедлив аналог (11). Докажем, что

$$
\int_{0}^{t} f(t-u) \mathrm{d} G(u)=\left\{\begin{array}{lll}
O\left(t^{-\beta-1} \ell_{F}(t)\right), & \text { если } & \beta \in(0,0.5], \\
\beta t^{-\beta-1} \ell_{F}(t)(1+o(1)), & \text { если } & \beta \in(0.5,1] .
\end{array}\right.
$$

Если $\beta \in(0,1)$, то соотношение (40) верно в силу леммы 5 из [1].

Если же $\beta=1$, то, фиксируя $\Delta>0$, полагая

$$
K:=\max \{k: k \Delta<t / 2\}, \Delta_{k}:=[(k-1) \Delta, k \Delta), t_{K}:=t-K \Delta
$$

и используя непрерывность функции $f(u)$, имеем

$$
\begin{aligned}
& \int_{0}^{t} f(t-u) \mathrm{d} G(u)=\int_{0}^{t_{K}} f(t-u) \mathrm{d} G(u)+\int_{t_{K}}^{t} f(t-u) \mathrm{d} G(u) \\
& \sim \ell_{F}(t) t^{-2}+\sum_{k=1}^{K} f\left(\theta_{\Delta_{k}}\right)(G(t-k \Delta)-G(t-(k-1) \Delta)),
\end{aligned}
$$

где $\theta_{\Delta_{k}} \in \Delta_{k}$ - значение, соответствующее классической оценке интеграла по $\Delta_{k}:=[t-(k-1) \Delta, t-k \Delta)$ от непрерывной функции. Последняя сумма оценивается величиной

$$
\left.O\left(\sum_{k=1}^{K} \frac{\ell_{F}(k \Delta)}{(k \Delta)^{2}}(G(t-(k-1) \Delta)-G(t-k) \Delta)\right)\right)=o\left(t^{-2} \min \{1, \ell(t)\}\right),
$$

что влечет (40) в случае $\beta=1$.

Напомним, что согласно леммам 1 и 2 верна оценка $1-H_{1}(t)=o\left(t^{-2}\right)$, а если $\beta=1$, то $H_{1}(t+\Delta)-H_{1}(t)=o\left(t^{-2} \min \{1, \ell(t)\}\right)$ при любых $\Delta>0$. Следовательно, в силу (21) эти же оценки верны при замене $1-H_{1}(t)$ на $\left(1-m_{11}\right)^{-1}-U_{m_{11}}(t)$. Кроме этого, плотности распределений $G_{2}(t)$ и $X_{1}(t)$ имеют порядок $O\left(t^{-\beta-1} \ell(t)\right)$ для $\beta \in(0,0.5]$ (в соответствии с (8) и (38)) и эквивалентны функции $\beta t^{-\beta-1} \ell(t)$ для $\beta \in(0.5,1]$ (в силу $(9)$ и $(38))$. Итак, свойства $v_{i j}(t)$ описаны. 
Учитывая последние свойства, для доказательства (24) вспомним (30) и отметим, что в представлении

$$
\int_{0}^{t} u_{X_{1}}(t-u) v_{i j}(u) \mathrm{d} u=\left(\int_{0}^{\sqrt{t}}+\int_{\sqrt{t}}^{t / 2}+\int_{t / 2}^{t}\right) u_{X_{1}}(t-u) \mathrm{d} V_{i j}(u)
$$

первый интеграл эквивалентен $D_{i j} \beta \Gamma(\beta) \mu_{2}^{-1}(t)$, второй оценивается величиной $o\left(\mu_{2}^{-1}(t)\right)$, а третий есть $O\left(t^{-1}\right)$ в силу (31) и (39). Теорема 2 доказана.

Покажем, что асимптотическое представление для $\left(\mathbf{u}^{c}(t)\right)^{\prime}$ (при условиях более жестких, чем Гипотеза $\mathbf{A}^{+}$) получается формальным дифференцированием правой части в (24). Для удобства приведем утверждение, объединяющее теорему 2 из [9] и теорему 3 из [8].

Теорема 5. Пусть распределение $G(t)$ удовлетворяет условию (34) и имеет абсолютно непрерывную плотность $g(t)$. Дополнительно предположим, что если $\beta \in(0,0.5]$, то найдется такая постоянная $C_{0}>0$, что

$$
\left|g^{\prime}(t)\right| \leqslant C_{0}(t+1)^{-\beta-2} \ell_{G}(t),
$$

а если $\beta \in(0.5,1]$, то $g^{\prime}(t)$ абсолютно непрерывна, $\left|g^{\prime \prime}(t)\right|$ интегрируема и вернъ соотношения $\mu_{G}(\infty)=\infty$,

$$
g^{\prime}(t) \sim(\beta+1) \beta t^{-\beta-2} \ell_{G}(t)
$$

$u$

$$
\operatorname{Var}_{u>t} u^{2} g^{\prime}(u) \sim C(\beta+1) \beta t^{-\beta} \ell_{G}(t)
$$

для некоторой постоянной $C \geqslant 1$.

Тогда если $\beta \in(0,1)$, то плотность $u_{G}(t)$ функции восстановления $U_{G}(t)$ дифферениируема $и$

$$
u_{G}^{\prime}(t) \sim(\beta-1) \beta \Gamma_{\beta} t^{-1} \mu_{G}^{-1}(t) .
$$

Если же $\beta=1$ и, кроме того, $\left|\ell_{G}^{\prime}(t)\right| t \in \mathcal{L}$ u для некоторой постоянной $C_{1} \geqslant 1$ выполняется соотношение

$$
\operatorname{Var}_{u>t}\left|\ell_{G}^{\prime}(u)\right| \sim C_{1}\left|\ell_{G}^{\prime}(t)\right|,
$$

mo

$$
u_{G}^{\prime}(t) \sim-\ell_{G}(t) t^{-1} \mu_{G}^{-2}(t) .
$$

Вернемся к исследованию свойств функции $\mathcal{N}_{\gamma}(t)$. По второй теореме о среднем для дифференцируемых функций

$$
\mathcal{N}_{\gamma}(t)-\mathcal{N}_{\gamma}(t-u)=u \mathcal{N}_{\gamma}^{\prime}(t-\theta u), \text { или } \frac{\mathcal{N}_{\gamma}(t)}{\mathcal{N}_{\gamma}(t-u)}=1+u \frac{\mathcal{N}_{\gamma}^{\prime}(t-\theta u)}{\mathcal{N}_{\gamma}(t-u)}
$$

для некоторого $\theta=\theta(t, u) \in[0,1]$ при всех $t, t-u \geqslant T_{0}$. Зафиксируем $p \in(0,1)$. Тогда в силу $(12)$ при $0 \leqslant u \leqslant t p$

$$
\frac{\mathcal{N}_{\gamma}^{\prime}(t-\theta u)}{\mathcal{N}_{\gamma}(t-u)}=\frac{\mathcal{N}_{\gamma}^{\prime}(t-\theta u)}{\mathcal{N}_{\gamma}(t-\theta u)} \frac{\mathcal{N}_{\gamma}(t-\theta u)}{\mathcal{N}_{\gamma}(t-u)} \sim \frac{\beta \gamma}{\beta+2} \frac{1}{t-\theta u}\left(\frac{t-\theta u}{t-u}\right)^{\beta \gamma /(\beta+2)} .
$$


Следовательно, существует такое $T_{1} \geqslant T_{0}$, что при $(1-p) t \geqslant T_{1}$ и $u \leqslant p t$

$$
\frac{\beta \gamma}{3 t} \leqslant \frac{\mathcal{N}_{\gamma}^{\prime}(t-\theta u)}{\mathcal{N}_{\gamma}(t-u)} \leqslant \frac{\beta \gamma}{2(t-\theta u)^{1-\beta \gamma /(\beta+2)}(t-u)^{\beta \gamma /(\beta+2)}} \leqslant \frac{\beta \gamma}{2(1-p) t} .
$$

Докажем следующее утверждение.

Лемма 3. Если $\beta \in(0,0.5]$ и для некоторого $\gamma \in(0,1]$ верно условие (13), то существуют такие постоянные $T_{2}>T_{1}>0$ и константа $c_{0}>0$, не зависящая om $c^{*}$, что при всех $t \geqslant T_{2}$

$$
G_{1} * H_{1}(t)-G_{1} * H_{1}\left(t-\mathcal{N}_{\gamma}(t)\right) \leqslant c_{0} c^{*} t^{-\beta-2} \ell(t) .
$$

Если $\beta \in(0.5,1)$ и для некоторого $\gamma \in(0, \min \{1,(1-\beta)(\beta+2) / \beta\}]$ справедливо условие (15), то

$$
G_{1} * H_{1}(t)-G_{1} * H_{1}\left(t-\mathcal{N}_{\gamma}(t)\right)=o\left(t^{-\beta-2} \ell(t)\right) .
$$

Замечание 1. Как и ранее, без ограничения общности будем предполагать, что $\ell(t) \in \mathcal{L}$ дифференцируема и $\ell^{\prime}(t)=o\left(\ell(t) t^{-1}\right)$. Более того, считаем, что функция $t^{-\beta} \ell(t)$ монотонно убывает при $t>1$ (см., например, [10], с. 25-26). Ввиду (12) очевидно, что чем меньше $\gamma$, тем более ограничительными являются условия (13) и (15). Это означает, что если $1 \leqslant(1-\beta)(\beta+2) / \beta$, или $\beta \in(0, \sqrt{3}-1]$, то условия (13) и (15) достаточно проверять (требовать выполнения в Гипотезе $\mathbf{A}^{++}$) только при $\gamma=1$, а если $\beta \in(\sqrt{3}-1,1)$, то при $\gamma=(1-\beta)(\beta+2) / \beta$.

Доказательство. Объединим доказательства соотношений (49) и (50). Для этого будем интерпретировать (15) как (13) с любыми $c^{*}>0$. Доказательство опирается на представление $(21)$ и оценку

$$
\begin{aligned}
& G_{1} * H_{1}(t)-G_{1} * H_{1}\left(t-\mathcal{N}_{\gamma}(t)\right) \\
\leqslant & \left(1-m_{11}\right) \sum_{s=1}^{s_{0}} m_{11}^{s-1}\left(G_{1}^{* s}(t)-G_{1}^{* s}\left(t-\mathcal{N}_{\gamma}(t)\right)+\left(1-m_{11}\right) \sum_{s=1+s_{0}}^{\infty} m_{11}^{s-1} .\right.
\end{aligned}
$$

Положим $s_{0}:=\left[-3.1 \ln t / \ln m_{11}\right] \sim c \ln t$, где $c:=-3.1 / \ln m_{11}$, тогда вторая сумма в (51) будет оцениваться величиной $o\left(t^{-\beta-2} \ell(t)\right)$ в силу того, что $m_{11}^{s_{0}}=o\left(t^{-\beta-2} \ell(t)\right)$. Для оценки первой суммы в (51) зафиксируем определяемую позже постоянную $\epsilon \in(0,1)$ и выберем $T_{2}(\epsilon)=T_{2} \geqslant T_{1}$ так, чтобы при $t \geqslant T_{2}$ и $u \leqslant(1-\epsilon) t$ было верно неравенство

$$
(t-u)^{-\beta-2} \ell(t-u) \leqslant \epsilon^{-3} t^{-\beta-2} \ell(t) .
$$

Так как $\ell(t) \in \mathcal{L}$ и $l^{\prime}(t)=o(\ell(t) / t)$, то найдется такая постоянная $C_{2}>0$, что

$$
(t-u)^{-\beta-2} \ell(t-u)-t^{-\beta-2} \ell(t) \leqslant C_{2} u t^{-\beta-3} \ell(t)
$$

для $t-u>\epsilon t$ и $t \geqslant T_{2}$. Положим $\mathrm{d}=\mathrm{d}_{\epsilon}:=1+0.5 \epsilon \beta \gamma(1-\epsilon)^{-1}$ и выберем такое $\epsilon \in(0,1)$, что $t^{\beta \gamma}>\mathrm{d}_{\epsilon}^{s_{0}}=\mathrm{d}_{\epsilon}^{c \ln t}$ при любом $t>1$. Заметим, что $\ln \mathrm{d}_{\epsilon} \sim 0.5 \epsilon \beta \gamma(1-\epsilon)^{-1}$ при $\epsilon \rightarrow+0$. Неравенство $t^{\beta \gamma}>\mathrm{d}_{\epsilon}^{s_{0}}$ будет верно при достаточно больших $t$, если $\ln t>0.5 c \epsilon(1-\epsilon)^{-1} \ln t$, или $2(1-\epsilon) / \epsilon>c$. Поэтому будем считать, что $0<\epsilon<$ $\min \{1 / 2,1 / c\}$. Положим

$$
C_{1}:=\left(3 \epsilon^{-3}+(1-\epsilon)^{-3}\right) \geqslant 32, y_{1}=y_{1}(t):=10 t^{\beta \gamma} \text { и } y_{k}=y_{k}(t):=y_{1} \mathrm{~d}_{\epsilon}^{1-k}
$$


при $k \in \mathbb{N}$. Заметим, что $y_{s_{0}}>1$. Выберем $T_{3} \geqslant T_{2}$ так, чтобы при $t \geqslant T_{3}, t-u>\epsilon t$, $1 \leqslant k \leqslant s_{0}$ и $y \in\left(1, y_{k}\right]$ выполнялось неравенство

$$
k y C_{1} c^{*}(t-u)^{-\beta-2} \ell(t-u)<1 .
$$

Отметим важный факт: постоянные $C_{1}, C_{2}$ могут зависеть от $\epsilon, \beta$ и $\gamma$, но если $\beta \in(0.5,1)$, то они не зависят от $k, y$ и $c^{*} \in(0,1)$.

Докажем по индукции, что при $t \geqslant T_{3}, 1 \leqslant k \leqslant s_{0}$ и $y \in\left[1, y_{k}\right]$ верно неравенство

$$
G_{1}^{* k}(t)-G_{1}^{* k}\left(t-y \mathcal{N}_{\gamma}(t)\right) \leqslant k y C_{1} c^{*} t^{-\beta-2} \ell(t):=\mathrm{f}_{k}(t, y),
$$

а затем применим (53) к первой сумме в (51). Заметим, что функция $\mathrm{f}_{k}(t, y)$ определена для достаточно больших $t$, что и нужно для оценок, но формально ее можно определить на всей полуоси следующим образом:

$$
\mathrm{f}_{k}(t, y)=\min \left\{1, k y C_{1} c^{*} t^{-\beta-2} \ell(t)\right\} .
$$

Для $t \geqslant T_{3}$ и $y \in\left(1, y_{1}\right]$ оценка $(13)$ с учетом неравенства $C_{1} \geqslant 32$ дает

$$
G_{1}(t)-G_{1}\left(t-y \mathcal{N}_{\gamma}(t)\right) \leqslant c^{*} y t^{-\beta-2} \ell(t) \leqslant \mathrm{f}_{1}(t, y) / 32,
$$

что доказывает (53) при $k=1$.

Напомним, что $l(t) \in \mathcal{L}$ можно выбрать таким образом, чтобы функции $t^{-\beta} \ell(t)$ и $t^{-\beta-2} \ell(t)$ монотонно убывали при $t \geqslant T_{3}$. При данном выборе функция $\mathrm{f}_{k}(t, y)$ также убывает при $t \geqslant T_{3}$ для любого натурального $k$. В соответствии с (52) при $t \geqslant T_{3}$ для $y \leqslant t, u \leqslant(1-\epsilon) t$ и $1 \leqslant k \leqslant s_{0} \leqslant c \ln t \leqslant t^{\beta}$ имеем

$$
\begin{gathered}
\mathrm{f}_{k}(t-u, y)=k y C_{1} c^{*}(t-u)^{-\beta-2} \ell(t-u) \leqslant \\
\leqslant k y C_{1} c^{*} t^{-\beta-2} \ell(t)+k y C_{1} c^{*} C_{2} u t^{-\beta-3} \ell(t)=\mathrm{f}_{k}(t, y)\left(1+C_{2} u / t\right) .
\end{gathered}
$$

При $t \geqslant T_{3}, u \leqslant(1-\epsilon) t$ и $k \leqslant s_{0}$ эта цепочка влечет оценку

$$
\mathrm{f}_{k}(t-u, y)-\mathrm{f}_{k}(t, y) \leqslant k y C_{1} c^{*} t^{-\beta-3} \ell(t) u C_{2} .
$$

Используя соотношения (53), (47), (48) и неравенство $2(1-\epsilon) \geqslant 1$ для $u \leqslant(1-\epsilon) t$, $y \in\left[1, y_{k} \mathrm{~d}_{\epsilon}^{-1}\right]=\left[1, y_{k+1}\right]$ при $1<k \leqslant s_{0}$, для достаточно больших $t$ получаем оценку

$$
\begin{aligned}
& G_{1}^{* k}(t-u)-G_{1}^{* k}\left(t-u-y \mathcal{N}_{\gamma}(t)\right) \\
= & G_{1}^{* k}(t-u)-G_{1}^{* k}\left(t-u-y \frac{\mathcal{N}_{\gamma}(t)}{\mathcal{N}_{\gamma}(t-u)} \mathcal{N}_{\gamma}(t-u)\right) \\
\leqslant & \mathrm{f}_{k}\left(t-u, y \frac{\mathcal{N}_{\gamma}(t)}{\mathcal{N}_{\gamma}(t-u)}\right) \leqslant \mathrm{f}_{k}\left(t-u, y\left(1+\frac{u \beta \gamma}{2(1-\epsilon) t}\right)\right) \\
\leqslant & \mathrm{f}_{k}(t-u, y(1+u \beta \gamma / t))=(1+u \beta \gamma / t) \mathrm{f}_{k}(t-u, y) .
\end{aligned}
$$

Заметим, что при $k=1, y \in\left[1, y_{2}\right]$ оценка (54) и последние выкладки дают:

$$
G_{1}(t-u)-G_{1}\left(t-u-y \mathcal{N}_{\gamma}(t)\right) \leqslant(1+u \beta \gamma / t) \mathrm{f}_{1}(t-u, y) / 32 .
$$

Используя определения, для $k \geqslant 1$ запишем неравенства

$$
G_{1}^{*(k+1)}(t)-G_{1}^{*(k+1)}\left(t-y \mathcal{N}_{\gamma}(t)\right) \leqslant J_{1}(k, t)+J_{2}(k, t)+J_{3}(k, t),
$$


где $y \in\left[1, y_{k+1}\right]$, a

$$
\begin{aligned}
J_{1}(k, t):= & \int_{0}^{(1-\epsilon) t}\left(G_{1}(t-u)-G_{1}\left(t-y \mathcal{N}_{\gamma}(t)-u\right)\right) \mathrm{d} G_{1}^{* k}(u), \\
J_{2}(k, t):= & \int_{0}^{\epsilon t}\left(G_{1}^{* k}(t-u)-G_{1}^{* k}\left(t-y \mathcal{N}_{\gamma}(t)-u\right)\right) \mathrm{d} G_{1}(u), \\
J_{3}(k, t):= & \left(G_{1}^{* k}((1-\epsilon) t)-G_{1}^{* k}\left((1-\epsilon) t-y \mathcal{N}_{\gamma}(t)\right)\right) \\
& \cdot\left(G_{1}(\epsilon t)-G_{1}\left(\epsilon t-y \mathcal{N}_{\gamma}(t)\right) .\right.
\end{aligned}
$$

По неравенству Чебышева

$$
\int_{t / \ln t}^{(1-\epsilon) t} \mathrm{~d} G_{1}^{* k}(u) \leqslant k \mu_{1} t^{-1} \ln t
$$

а в силу (55) при $u \in[0, t / \ln t]$

$$
\mathrm{f}_{1}(t-u, y)-\mathrm{f}_{1}(t, y) \leqslant C_{2} \mathrm{f}_{1}(t, y) / \ln t .
$$

Отсюда, используя неравенства (57), для всех $1 \leqslant k \leqslant s_{0} \leqslant c \ln t$ при $t>T_{3}$, $1+u \beta \gamma / t<2$ и $y \in\left[1, y_{k+1}\right]$ имеем

$$
\begin{aligned}
& J_{1}(k, t) \leqslant \int_{0}^{(1-\epsilon) t} \mathrm{f}_{1}(t-u, y) \mathrm{d} G_{1}^{* k}(u) / 16 \leqslant \mathrm{f}_{1}(t, y) / 8 \\
& J_{3}(k, t) \leqslant 2 \mathrm{f}_{k}(t(1-\epsilon), y) \mathrm{f}_{1}(\epsilon t, y) / 8 \leqslant \mathrm{f}_{1}(t, y) / 8
\end{aligned}
$$

Учитывая дополнительно неравенства (55) и (56), получаем

$$
J_{2}(1, t) \leqslant \int_{0}^{\epsilon t}(1+u \beta \gamma / t) \mathrm{f}_{k}(t-u, y) \mathrm{d} G_{1}(u)=(1+o(1)) \mathrm{f}_{k}(t, y) .
$$

В результате представление (58) и следующие за ним оценки приводят к (53) для $k+1 \leqslant s_{0}$, если оно было верно для $k$, что по индукции доказывает (53) для всех $k \leqslant s_{0}$.

Это совместно с (51) и определением $s_{0}$ влечет

$$
G_{1} * H_{1}(t)-G_{1} * H_{1}\left(t-\mathcal{N}_{\gamma}(t)\right) \leqslant\left(1-m_{11}\right) \sum_{s=1}^{s_{0}} m_{11}^{s-1} s C_{1} c^{*} t^{-\beta-2} \ell(t)+\psi(t),
$$

где $\psi(t)=o\left(t^{-\beta-2} \ell(t)\right)$, и завершает доказательство леммы 3.

Лемма 4. Пусть выполнено условие (18), тогда для того же $\Delta>0$ верна оценка

$$
G_{1} * H_{1}(t+\Delta)-G_{1} * H_{1}(t)=o\left(t^{-3} \min \{1, \ell(t)\}\right) .
$$

Доказательство. Необходимо почти дословно повторить предшествующее доказательство с модификациями, которые мы укажем.

В интересующем нас случае представление (51) для $G_{1} * H_{1}(t+\Delta)-G_{1} * H_{1}(t)$ принимает вид

$$
\left(1-m_{11}\right) \sum_{s=1}^{s_{0}} m_{11}^{s-1}\left(G_{1}^{* s}(t+\Delta)-G_{1}^{* s}(t)\right)+o\left(t^{-3} \min \{1, \ell(t)\}\right),
$$


где $s_{0}=\left[-3.1 \ln (t) / \ln \left(m_{11}\right)\right]$ и $\Delta>0$ - произвольное фиксированное число.

Если в доказательстве леммы 3 выбрать сколь угодно малое $c^{*}>0$ и заменить $\ell(t)$, $y, \mathcal{N}_{\gamma}(t)$ и $\beta$ на $\min \{1, \ell(t)\}, 1, \Delta>0$ и 1 , соответственно, то получим $d=y_{k} \equiv 1$, а $\mathrm{f}_{k}(t, 1)=k C_{1} c^{*} t^{-3} \min \{1, \ell(t)\}$. Подстановка указанных значений в цепочку оценок, использованных при доказательстве леммы 3 , приводит к доказательству леммы 4 .

Если же повторить подстановку из предшествующего абзаца, а вместо $\beta$ выбрать не 1 , а 0 , то получим $\mathrm{f}_{k}(t, 1)=k C_{1} c^{*} t^{-2} \min \{1, \ell(t)\}$, и доказательство леммы 4 превращается в доказательство леммы 2.

Доказательство теоремы 3. Если верна Гипотеза $\mathbf{A}^{++}$, то функция распределения $X_{1}(t)$, определенная в $(22)$, имеет асимптотику $1-X_{1}(t) \sim 1-G_{2}(t)$ в силу (33) и, следовательно, может быть представлена в виде $X_{1}(t)=t^{-\beta} \ell_{X_{1}}(t)$, где $\ell_{X_{1}}(t) \in \mathcal{L}$ абсолютно непрерывна, а ее производная $x_{1}(t)$, приведенная в (38), дифференцируема и

$$
\begin{aligned}
& x_{1}^{\prime}(t)=m_{22} g_{2}^{\prime}(t)+\left(1-m_{22}\right) g_{2}^{\prime} * G_{1} * H_{1}(t), \text { если } \beta \in(0,1], \\
& x_{1}^{\prime \prime}(t)=m_{22} g_{2}^{\prime \prime}(t)+\left(1-m_{22}\right) g_{2}^{\prime \prime} * G_{1} * H_{1}(t), \text { если } \beta \in(0.5,1] .
\end{aligned}
$$

Проверим, что условия теоремы 5 верны для $G(t)=X_{1}(t)$, путем переноса свойств $G_{2}(\mathrm{t})$ из Гипотезы $\mathbf{A}^{++}$на $X_{1}(t)$. При $\beta \in(0,1)$ положим $K:=\max \left\{k: k \mathcal{N}_{\gamma}(t / 2)<\right.$ $t / 2\}, \Delta_{k}:=\left[(k-1) \mathcal{N}_{\gamma}(t / 2), k \mathcal{N}_{\gamma}(t / 2)\right), t_{K}:=t-K \mathcal{N}_{\gamma}(t / 2)$, а при $\beta=1$ данные величины определены в (41).

Ввиду условий (14) и (16) при $\beta \in(0.5,1]$ величина $\sup _{u \in \Delta_{k}} g_{2}^{\prime}(u)$ ограничена для $k=1$, а для $k>1$ имеет порядок $O\left(\left((k-1) \mathcal{N}_{\gamma}(t)\right)^{-\beta-2} \ell\left(k \mathcal{N}_{\gamma}(t)\right)\right)$, если $\beta \in(0,1)$, и порядок $O\left(((k-1) z)^{-3} \ell(k z)\right)$, если $\beta=1$. Леммы 3 и 4 и условие (4) позволяют оценить интеграл $\int_{u \in \Delta_{k}} d G_{1} * H_{1}(t-u)$ сверху функциями порядка $O\left(t^{-\beta-2} \ell(t)\right)$, если $\beta \in(0,0.5]$, порядка $o\left(t^{-\beta-2} \ell(t)\right)$, если $\beta \in(0.5,1)$, и порядка $o\left(t^{-3} \min \{1, \ell(t)\}\right)$, если $\beta=1$.

В итоге получаем

$$
\int_{0}^{t_{K}} g_{2}^{\prime}(u) \mathrm{d}\left(G_{1} * H_{1}(t-u)\right)=\left\{\begin{array}{lll}
O\left(t^{-\beta-2} \ell(t)\right), & \text { если } & \beta \in(0,0.5], \\
o\left(t^{-\beta-2} \ell(t)\right), & \text { если } & \beta \in(0.5,1] .
\end{array}\right.
$$

При $\beta \in(0,0.5]$ верна оценка

$$
g_{2}^{\prime} * G_{1} * H_{1}(t)=\left(\int_{0}^{t_{K}}+\int_{t_{K}}^{t}\right) g_{2}^{\prime}(u) \mathrm{d}\left(G_{1} * H_{1}(t-u)\right)=O\left(t^{-\beta-2} \ell(t)\right),
$$

где мы воспользовались (61), а для второго интеграла нужно использовать те же аргументы, что и выше.

Поскольку верны оценки $\int_{u \in(t-N, t]} g_{2}^{\prime}(u) \mathrm{d}\left(G_{1} * H_{1}(t-u)\right) \sim g_{2}^{\prime}(t) G_{1} * H_{1}(N)$ и $\int_{u \in\left(t_{K}, t-N\right)} g_{2}^{\prime}(u) \mathrm{d}\left(G_{1} * H_{1}(t-u)\right)=O\left(g_{2}^{\prime}(t)\left(1-G_{1} * H_{1}(N)\right)\right)$ при $\beta \in(0.5,1]$ и произвольном $N>0$, то аналогом (62) будет соотношение

$$
g_{2}^{\prime} * G_{1} * H_{1}(t) \sim g_{2}^{\prime}(t) .
$$

Из представления (59) и оценки (62) следует, что $H_{1}(t)$ при $\beta \in(0,0.5]$ удовлетворяет условиям теоремы 5 .

Убедимся теперь, что условия второй части теоремы 5 (в случае $\beta \in(0.5,1])$ выполнены для $G(t)=X_{1}(t)$. Представления (59), (60) и (21) при выполнении 
Гипотезы $\mathbf{A}^{++}$влекут абсолютную непрерывность $x_{1}^{\prime}(t)$, что в совокупности с конечностью первого момента для $G_{1} * H_{1}(t)$ обеспечивает интегрируемость $\left|x_{1}^{\prime \prime}(t)\right|$ и справедливость равенства $\mu_{X_{1}}(\infty)=\infty$. Наконец, из соотношений (59) и (63) получаем, что для $X_{1}(t)$ выполнено условие (42). Аргументы о представлении функции ограниченной вариации через разность монотонных, следующие за (38), естественно приводят к доказательству (43). Поэтому теорема 5 при выполнении Гипотезы $\mathbf{A}^{++}$ в случае $\beta \in(0,1)$ влечет

$$
u_{X_{1}}^{\prime}(t) \sim(\beta-1) \beta \Gamma(\beta) t^{-1} \mu_{2}^{-1}(t) .
$$

Осталось проверить выполнение условий теоремы 5 для $X_{1}(t)$, если $\beta=1$ и верна Гипотеза $\mathbf{A}^{++}$, то есть соотношения $\left|\ell_{X_{1}}^{\prime}(t)\right| t \in \mathcal{L}$ и (45). Тогда из представления (46) будет следовать цепочка

$$
u_{X_{1}}^{\prime}(t) \sim \ell(t) t^{-1} \mu_{2}^{-2}(t) \sim \ell(t) t^{-1} \ell_{1}^{-2}(t) .
$$

В итоге, используя тождество $\left(u_{i j}^{c}(t)\right)^{\prime}=v_{i j} * u_{X_{1}}^{\prime}(t)$, соотношения $(64)$ и $(65)$ и повторяя почти дословно аргументы из доказательства теоремы 2 , выводим основную часть теоремы 3 - формулы $(25)$ и $(26)$.

Приступим к проверке того, что $\left|\ell_{X_{1}}^{\prime}(t)\right| t \in \mathcal{L}$, если $\beta=1$ и верна Гипотеза $\mathbf{A}^{++}$. Перепишем (22) в виде

$$
\ell_{X_{1}}(t)=\ell(t)-\left(1-m_{22}\right) t G_{2} *\left(1-G_{1} * H_{1}(\cdot)\right)(t),
$$

тогда вычитаемое в правой части последнего соотношения (без сомножителя $\left.\left(1-m_{22}\right)\right)$ можно представить так:

$$
\int_{0}^{t}\left(1-G_{1} * H_{1}(u)\right) g_{2}(t-u)(t-u) \mathrm{d} u+\int_{0}^{t} G_{2}(t-u) u \mathrm{~d}\left(1-G_{1} * H_{1}(u)\right),
$$

а производная от данного выражения при разбиении областей интегрирования пополам с учетом того, что $\left(t g_{2}(t)\right)^{\prime} \sim-g_{2}(t)$, по леммам 2 и 4 оценивается выражением

$$
o\left(t^{-2} \min \{1, \ell(t)\}\right)+O\left(g_{2}(t)\right)+O\left(g_{2}(t)\right)+o\left(t^{-2} \min \{1, \ell(t)\}\right)=t^{-2} O(1+\ell(t)) .
$$

Поэтому функция $\left|\ell_{X_{1}}^{\prime}(t) t\right| \sim\left|\ell^{\prime}(t) t\right|$ будет медленно меняющейся. Соотношение (45) для $G=X_{1}$ получается немедленно из представлений (66) и (67) и следующей за ними оценки.

Наконец, вспоминая определение $U_{i 1}^{d}(t)$ из $(23)$ и применяя оценки (13) и (49), легко убедиться в справедливости (27) и, обращаясь к лемме 4, получить (28).

\section{3. Применения для вывода асимптотик моментов ветвящегося процесса}

Введем условные средние $\mathbf{E}_{i}(\cdot):=\mathbf{E}\left\{\cdot \mid \mathbf{Z}(0)=\delta_{i}\right\}$, где $\delta_{1}=(1,0)^{T}, \delta_{2}=(0,1)^{T}$. Приведем ряд определений и утверждений из раздела $3_{[1]}$ (без доказательств) об асимптотике моментов $\mathbf{E}_{i} Z_{j}(t)=: P_{i j}(t)$ и $\mathbf{E}_{i} Z_{j}^{2}(t)=: P_{i j}^{(2)}(t)$ для критических процессов Беллмана-Харриса с двумя типами частиц, удовлетворяющих условиям (1)-(5) с $\beta \in(0,1]$, а затем, основываясь на результатах из предшествующего раздела, докажем ряд более точных утверждений о свойствах моментов. 
Обозначим

$$
\begin{gathered}
\mathbf{G}_{\mathbf{I}}(t)=\left(G_{i}(t) \delta_{i j}\right)_{i, j=1}^{2}, \mathbf{P}(t):=\left(P_{i j}(t)\right)_{i, j=1}^{2}, \mathbf{P}^{(2)}(t):=\left(P_{i j}^{(2)}(t)\right)_{i, j=1}^{2}, \\
\mathbf{P}_{i}(t):=\left(P_{1 i}(t), P_{2 i}(t)\right)^{T}, \mathbf{P}_{i}^{(2)}(t):=\left(P_{1 i}^{(2)}(t), P_{2 i}^{(2)}(t)\right)^{T} .
\end{gathered}
$$

Для векторов $\mathbf{x}=\left(x_{1}, x_{2}\right)^{T}$ и $\mathbf{y}=\left(y_{1}, y_{2}\right)^{T}$ положим $\mathbf{x} \otimes \mathbf{y}=\left(x_{1} y_{1}, x_{2} y_{2}\right)^{T}$. Как показано в [1] (см. формулы $(3.5)_{[1]},(3.7)_{[1]},(3.8)_{[1]}$ и $\left.(3.9)_{[1]}\right)$,

$$
\begin{aligned}
& \mathbf{P}(t)=\mathbf{U} *\left(\mathbf{I}-\mathbf{G}_{\mathbf{I}}(\cdot)\right)(t), \\
& \mathbf{P}^{(2)}(t)=\mathbf{P}(t)+\mathbf{U} * \mathbf{T}(t),
\end{aligned}
$$

где $\mathbf{T}(t):=\left(T_{k j}(t)\right)_{k, j=1}^{2}-$ матрица с элементами

$$
\begin{array}{r}
T_{j k}(t)=\sum_{l, m=1}^{2} b_{l m}^{j} \int_{0}^{t} P_{l k}(t-u) P_{m k}(t-u) \mathrm{d} G_{j}(u), \\
\mathbf{P}_{i}(t)=\mathbf{U} *\left(\delta_{i} \otimes(\mathbf{1}-\mathbf{G}(\cdot))\right)(t), \quad i=1,2,
\end{array}
$$

при этом последнее соотношение является эквивалентной записью (68). Это позволяет исследовать свойства векторов $\left(P_{11}(t), P_{21}(t)\right)$ и $\left(P_{12}(t), P_{22}(t)\right)$ независимо друг от друга. Напомним лемму 16 из [1].

Лемма 5. Если верна Гипотеза $\boldsymbol{A}$, то

$$
\mathbf{P}(t) \sim\left(\begin{array}{cc}
D_{11} \frac{\mu_{1} \beta \Gamma_{\beta}}{\mu_{2}(t)} & D_{12} \\
D_{21} \frac{\mu_{1} \beta \Gamma_{\beta}}{\mu_{2}(t)} & D_{22}
\end{array}\right)=\mathbf{D}\left(\begin{array}{cc}
\frac{\mu_{1} \beta \Gamma_{\beta}}{\mu_{2}(t)} & 0 \\
0 & 1
\end{array}\right) .
$$

Изучим детальнее представление (70). Для этого запишем $\mathbf{P}(t)$ как сумму $\mathbf{P}(t)=$ $\mathbf{P}^{d}(t)+\mathbf{P}^{c}(t)$, где

$$
\begin{aligned}
& \mathbf{P}^{d}(t)=\left(P_{i j}^{d}(t)\right)_{i, j=1}^{2}:=\mathbf{U}^{d} *\left(\mathbf{I}-\mathbf{G}_{\mathbf{I}}(\cdot)\right)(t), \\
& \mathbf{P}^{c}(t)=\left(P_{i j}^{c}(t)\right)_{i, j=1}^{2}:=\mathbf{U}^{c} *\left(\mathbf{I}-\mathbf{G}_{\mathbf{I}}(\cdot)\right)(t),
\end{aligned}
$$

и ужесточим ограничения на $G_{1}(t)$ и $G_{2}(t)$.

Теорема 6. Если справедлива Гипотеза $\boldsymbol{A}^{++}$, то функции $P_{i 1}(t), i=1,2$, монотонны при достаточно больших $t$ и верны оценки

$$
P_{i 1}^{d}(t)-P_{i 1}^{d}\left(t-\mathcal{N}_{\gamma}(t)\right)=\left\{\begin{array}{lll}
O\left(t^{-\beta-2} \ell(t)\right), & \text { если } & \beta \in(0,0.5] \\
o\left(t^{-\beta-2} \ell(t)\right), & \text { если } & \beta \in(0.5,1),
\end{array}\right.
$$

а при $\beta=1$ илюбъх $\Delta>0$

$$
P_{i 1}^{d}(t)-P_{i 1}^{d}(t-\Delta)=o\left(t^{-3} \ell(t)\right)
$$

Компоненты $P_{i j}^{c}(t), i, j=1,2$, абсолютно непрерывны и при $\beta \in(0,1)$

$$
P_{i 1}^{c \prime}(t) \sim-D_{i 1} \mu_{1} \beta(1-\beta) \Gamma_{\beta} t^{-1} \mu_{2}^{-1}(t)
$$


а при $\beta=1$

$$
P_{i 1}^{c \prime}(t) \sim-D_{i 1} \mu_{1} \ell(t) t^{-1} \ell_{1}^{-2}(t) .
$$

Далее,

$$
P_{i 2}^{c \prime}(t)=o\left(t^{-1}\right)
$$

если $\beta \in(0,1), u$

$$
P_{i 2}^{c \prime}(t)=O(1) t^{-1} \ell(t) \ell_{1}^{-1}(t)=o\left(t^{-1}\right)
$$

если $\beta=1$.

$B$ случае выполнения условия $\int_{1}^{\infty} t^{-1} \ell(t) \ell_{1}^{-1}(t) d t=\infty$ оценку $O(1)$ в (76) можно заменить на о(1).

Доказательство. При $\beta \in(0,1)$

$$
\begin{gathered}
P_{i 1}^{d}(t)-P_{i 1}^{d}\left(t-\mathcal{N}_{\gamma}(t)\right)=\int_{t-\mathcal{N}_{\gamma}(t)}^{t}\left(1-G_{1}(t-y)\right) \mathrm{d} U_{i 1}^{d}(y) \\
-\int_{0}^{t-\mathcal{N}_{\gamma}(t)}\left(G_{1}(t-y)-G_{1}\left(t-\mathcal{N}_{\gamma}(t)-y\right)\right) \mathrm{d} U_{i 1}^{d}(y),
\end{gathered}
$$

а при $\beta=1$

$$
\begin{gathered}
P_{i 1}^{d}(t)-P_{i 1}^{d}(t-\Delta)=\int_{t-\Delta}^{t}\left(1-G_{1}(t-y)\right) \mathrm{d} U_{i 1}^{d}(y) \\
-\quad \int_{0}^{t-\Delta}\left(G_{1}(t-y)-G_{1}(t-\Delta-y)\right) \mathrm{d} U_{i 1}^{d}(y) .
\end{gathered}
$$

По теореме 3

$$
\begin{aligned}
& \int_{t-\mathcal{N}_{\gamma}(t)}^{t}\left(1-G_{1}(t-y)\right) \mathrm{d} U_{i 1}^{d}(y) \\
\leqslant & U_{i 1}^{d}(t)-U_{i 1}^{d}\left(t-\mathcal{N}_{\gamma}(t)\right)=\left\{\begin{array}{lll}
O\left(t^{-\beta-2} \ell(t)\right), & \text { если } & \beta \in(0,0.5], \\
o\left(t^{-\beta-2} \ell(t)\right), & \text { если } & \beta \in(0.5,1),
\end{array}\right.
\end{aligned}
$$

а при $\beta=1$

$$
U_{i 1}^{d}(t)-U_{i 1}^{d}(t-\Delta)=o\left(t^{-3} \ell(t)\right) .
$$

Заметим, что оценки (49) и (50) являются аналогом (27). Поэтому аналогом оценок $(61)-(63)$ для $g_{2}^{\prime} * H_{1}(t)$ будет соотношение

$$
\begin{aligned}
& \int_{0}^{t-\mathcal{N}_{\gamma}(t)}\left(G_{1}(t-y)-G_{1}\left(t-\mathcal{N}_{\gamma}(t)-y\right)\right) \mathrm{d} U_{i 1}^{d}(y) \\
= & \left\{\begin{array}{lll}
O\left(t^{-\beta-2} \ell(t)\right), & \text { если } & \beta \in(0,0.5], \\
o\left(t^{-\beta-2} \ell(t)\right), & \text { если } & \beta \in(0.5,1) .
\end{array}\right.
\end{aligned}
$$

Это доказывает (71). Теперь, следуя схеме доказательства (28) из теоремы 3 , легко вывести оценку (72).

Далее, по определению и ввиду того, что $u_{i j}^{c}(0)=0$, получаем

$$
\begin{aligned}
P_{i j}^{c}(t) & =-\int_{0}^{t}\left(1-G_{j}(y)\right) u_{i j}^{c}(t-y) \mathrm{d} y, \\
P_{i j}^{c \prime}(t) & =-\int_{0}^{t}\left(1-G_{j}(y)\right) u_{i j}^{c}{ }^{\prime}(t-y) \mathrm{d} y .
\end{aligned}
$$


По теореме 3 для $j=1$ и любого $\delta \in(0,1)$ имеем

$$
\begin{aligned}
& \int_{0}^{t^{\delta}}\left(1-G_{1}(y)\right) u_{i 1}^{c \prime}(t-y) \mathrm{d} y \\
\sim & D_{i 1} \int_{0}^{t^{\delta}}\left(1-G_{1}(y)\right) \mathrm{d} y \cdot\left\{\begin{array}{lll}
\beta(\beta-1) \Gamma_{\beta} t^{-1} \mu_{2}^{-1}(t), & \text { если } & \beta \in(0,1), \\
-\ell(t) t^{-1} \ell_{1}^{-2}(t), & \text { если } & \beta=1 .
\end{array}\right.
\end{aligned}
$$

Если $\beta=1$, то, учитывая (4) и (11), для любого $\epsilon \in(0,1)$ имеем

$$
\int_{t \epsilon}^{t}\left(1-G_{1}(y)\right) u_{i 1}^{c}{ }^{\prime}(t-y) \mathrm{d} y=o\left(\min \left\{t^{-2}, t^{-\beta-1} \ell(t)\right\}\right) .
$$

Наконец, найдется такое $C>0$, что для $y \in[0, t \epsilon]$ верно неравенство $\left(u_{i 1}^{c}(t-y)\right)^{\prime} \leqslant$ $C\left(u_{i 1}^{c}(t)\right)^{\prime}$, что влечет

$$
\int_{t^{\delta}}^{t \varepsilon}\left(1-G_{1}(y)\right)\left(u_{i 1}^{c}(t-y)\right)^{\prime} \mathrm{d} y \leqslant C\left(u_{i 1}^{c}(t)\right)^{\prime} \int_{t^{\delta}}^{\infty}\left(1-G_{1}(y)\right) \mathrm{d} y=o\left(\left(u_{i 1}^{c}(t)\right)^{\prime}\right) .
$$

В итоге получаем оценки (73) и (74).

Отметим, что по определению $P_{i 1}^{d}(t)$ является разностью двух монотонных функций, которые по теоремам 3 и 6 на интервалах длины $\Delta>0$ будут иметь приращения порядка $O\left(t^{-\beta-2} \ell(t)\right)$. Функции $P_{i 1}^{c}(t)$ при больших $t$ имеют отрицательные производные, т.е. монотонны. Поэтому монотонность $P_{i 1}(t)$ при достаточно больших $t$ следует из оценок $(73),(74)$ и

$$
\begin{aligned}
P_{i 1}(t+\Delta)-P_{i 1}(t) & =P_{i, 1}^{d}(t+\Delta)-P_{i 1}^{d}(t)+P_{i 1}^{c}(t+\Delta)-P_{i 1}^{c}(t) \\
& =O\left(t^{-\beta-2} \ell(t)\right)+\Delta(1+o(1))\left(P_{i 1}^{c}(t)\right)^{\prime},
\end{aligned}
$$

а также соотношения $t^{-\beta-2} \ell(t)=o\left(\left(t \mu_{2}(t)\right)^{-1}\right)$.

При $j=2$ воспользуемся представлением

$$
\begin{aligned}
P_{i 2}^{c \prime}(t) & =-\int_{0}^{t}\left(G_{2}(t)-G_{2}(t-y)\right) u_{i 2}^{c}{ }^{\prime}(y) \mathrm{d} y-\left(1-G_{2}(t)\right) \int_{0}^{t} u_{i 2}^{c \prime}(y) \mathrm{d} y \\
& =: \quad I_{P 1}(t)+I_{P 2}(t) .
\end{aligned}
$$

Прежде всего, из $(5)$, Гипотезы $\mathbf{A}^{++}$и $(25)$ для любого $\epsilon \in(0,1)$ получаем, что

$$
\begin{aligned}
& \left(G_{2}(t)-G_{2}(t-y)\right) u_{i 2}^{c \prime}(y) \\
& = \begin{cases}O(1) t^{-\beta-1} \ell(t) u_{i 2}^{c}(y), & \text { если } y \in(0, \epsilon t), \\
(1+o(1)) D_{i 2} \beta(1-\beta)^{2} \Gamma_{\beta}\left(t^{-\beta}-(t-y)^{-\beta}\right) y^{\beta-2}, & \text { если } y \in(\epsilon t,(1-\epsilon) t), \\
O(1)\left(1-G_{2}(t-y)\right) \ell^{-1}(t) t^{\beta-2}, & \text { если } y \in((1-\epsilon) t, t) .\end{cases}
\end{aligned}
$$

Отсюда, полагая $\mathcal{I}_{\epsilon}:=\int_{\epsilon}^{1-\epsilon}\left((1-v)^{-\beta}-1\right) v^{\beta-2} \mathrm{~d} v$, выводим, что

$$
I_{P 1}(t)=\epsilon^{\beta} O(1) t^{-1}+t^{-1} D_{i 2} \beta(1-\beta)^{2} \Gamma_{\beta} \mathcal{I}_{\epsilon}(1+o(1))+\epsilon^{1-\beta} O(1) t^{-1} .
$$

Последнее совместно с определением $\mathcal{I}:=\lim _{\epsilon \rightarrow+0} \mathcal{I}_{\epsilon} \in(0, \infty)$ приводит к оценке

$$
I_{P 1}(t) \sim t^{-1} D_{i 2} \beta(1-\beta)^{2} \Gamma_{\beta} \mathcal{I} .
$$


Далее, в силу (5) и теоремы 3

$$
I_{P 2}(t)=-\left(1-G_{2}(t)\right) u_{i 2}^{c}(t) \sim-t^{-1} D_{i 2} \beta(1-\beta) \Gamma_{\beta} .
$$

В итоге, $(1-\beta) \mathcal{I}=1$, ибо, если это не так, то $P_{i 2}^{c}{ }^{\prime}(t) \sim c t^{-1}$ для некоторого конечного $c \neq 0$, что противоречит (70). Соотношение (75) доказано.

$\mathrm{B}$ случае $\beta=1$ естественная модификация предшествующих оценок приводит к равенству

$$
I_{P 1}(t)=t^{-1} \ell(t) \ell_{1}^{-1}(t)\left(\epsilon O(1) \ell(t) \ell_{1}^{-1}(t)+D_{i 2} \mathcal{I}_{\epsilon}(1+o(1))+O(1) \ell(t) \ell_{1}^{-1}(t)\right)
$$

и асимптотическому соотношению

$$
I_{P 1}(t) \sim D_{i 2} t^{-1} \ell(t) \ell_{1}^{-1}(t) \mathcal{I} .
$$

Последнее совместно с $I_{P 2} \sim-D_{i 2} t^{-1} \ell(t) \ell_{1}^{-1}(t)$ (что следует из условия (5) и теоремы 3) дает $P_{i 2}^{c}(t)=(c+o(1)) t^{-1} \ell(t) \ell_{1}^{-1}(t)$ для некоторого конечного $c$. По определению $P_{i 2}^{c}(t)-P_{i 2}^{c}(0)=\int_{0}^{t} P_{i 2}^{c}(t)$, но $P_{i 2}(t) \rightarrow D_{i 2}$ по лемме 5 , следовательно, $c=0$, если $\int_{1}^{\infty} t^{-1} \ell(t) \ell_{1}^{-1}(t) d t=\infty$. Соотношение (76) со следующим за ним замечанием, а, в итоге, и теорема 6 доказаны.

Найдем грубые оценки сверху и снизу для элементов матрицы $\mathbf{P}^{(2)}(t)$. Определим постоянные

$$
\begin{array}{r}
D_{l m}^{*}:=\int_{0}^{\infty} P_{l 1}(u) P_{m 1}(u) \mathrm{d} u\left(\text { конечны только при } \int_{0}^{\infty} \mu_{2}^{-2}(t) \mathrm{d} t<\infty\right), \\
S_{j}^{*}:=\sum_{l, m=1,2} b_{l m}^{j} D_{l m}^{*}, S_{j k}:=\left(\left(\mu_{1} \beta \Gamma_{\beta}\right)^{2} 1_{\{k=1\}}+1_{\{k=2\}}\right) \sum_{l, m=1,2} b_{l m}^{j} D_{l k} D_{m k},
\end{array}
$$

где $l, m, j, k=1,2$, и докажем результат, существенно уточняющий лемму 18 из [1] в области основных допущений.

Лемма 6. Пусть верна Гипотеза $\boldsymbol{A}$. Тогда при $j=1,2$

$$
T_{j 2}(t) \sim S_{j 2} .
$$

Пусть, кроме того,

i) $д л я \beta \in(1 / 2,2 / 3)$ верны оченки

$$
G_{2}(t+z)-G_{2}(t)=o\left(t^{\phi(\beta)-\delta}\right),
$$

где $\phi(\beta)=-\beta-3 / 2+(2-2 \beta)^{-1}, a z>0 u \delta>0-$ любые фиксированные;

ii) $\partial \Omega я \beta=2 / 3$

$$
G_{2}\left(t+\ell_{\#}^{-2}(t)\right)-G_{2}(t)=o\left(\left(1-G_{2}(t)\right) \ell(t)\right),
$$

где $\ell_{\#}(t)=\min (1, \ell(t))$.

Тогда выполняются соотношения

$$
T_{j 1}(t)=\left\{\begin{array}{lll}
O\left(t^{-\beta-1} \ell(t)\right), & \text { если } & \beta \in(0,1 / 3), \\
\frac{9 S_{j 1}+o(1)}{4 t^{4 / 3} \ell^{2}(t)}+O\left(t^{-4 / 3} \ell(t)\right), & \text { если } & \beta=1 / 3, \\
\frac{S_{j 1}+o(1)}{\mu_{2}^{2}(t)}, & \text { если } & \beta \in(1 / 3,1] .
\end{array}\right.
$$


Оценку $O\left(t^{-\beta-1} \ell(t)\right)$ при $\beta \in(0,1 / 3]$ в (80) можно заменить на $\asymp t^{-\beta-1} \ell(t)$ или на $\beta\left(S_{j}^{*}+o(1)\right) t^{-\beta-1} \ell(t)$, если условие (7) в первом случае заменить условием: найдутся такие постоянные $C>c$ и $T_{0}$, что при $t \geqslant T_{0}$ и для любого $\Delta>0$ вернъ оченки

$$
c \Delta \ell(t) t^{-\beta-1} \leqslant G_{2}(t+\Delta)-G_{2}(t) \leqslant C \Delta \ell(t) t^{-\beta-1},
$$

а во втором случае заменить условием: для любого $\Delta>0$ выполнено соотношение

$$
G_{2}(t+\Delta)-G_{2}(t) \sim \beta \Delta \ell(t) t^{-\beta-1}
$$

Доказательство. Как следует из (69), для проверки (77) достаточно показать, что при $l, m, j=1,2$

$$
\begin{aligned}
& \left(P_{l j}(\cdot) P_{m j}(\cdot)\right) * G_{1}(t) \sim P_{l j}(t) P_{m j}(t), \\
& \left(P_{l 2}(\cdot) P_{m 2}(\cdot)\right) * G_{2}(t) \sim P_{l 2}(t) P_{m 2}(t) \sim D_{l 2} D_{m 2} .
\end{aligned}
$$

Для вывода (83) зафиксируем произвольное $\epsilon \in(0,1)$ и разделим область интегрирования на две части: от 0 до $\epsilon t$ и от $\epsilon t$ до $t$, а затем воспользуемся условием (4) и леммой 5 . Так как $\epsilon \in(0,1)$, то, выбирая его сколь угодно малым и учитывая стандартные свойства правильно меняющихся функций, легко получить требуемый результат. Соотношение (84) немедленно следует из асимптотики второго столбца в (70).

Если верна Гипотеза А и выполнены условия і) и іi), то ввиду (83) соотношение (80) будет следовать из оценок

$$
\left(P_{l 1}(\cdot) P_{m 1}(\cdot)\right) * G_{2}(t)=\left\{\begin{array}{l}
O\left(t^{-\beta-1} \ell(t)\right), \\
\frac{\left(\mu_{1} \Gamma_{1 / 3}\right)^{2} D_{l 1} D_{m 1}+o(1)}{4 t^{4 / 3} l^{2}(t)}+O\left(t^{-4 / 3} \ell(t)\right), \\
\frac{\left(\mu_{1} \beta \Gamma_{\beta}\right)^{2} D_{l 1} D_{m 1}+o(1)}{\mu_{2}^{2}(t)}
\end{array}\right.
$$

справедливых для $\beta \in(0,1 / 3), \beta=1 / 3$ и $\beta \in(1 / 3,1]$, соответственно. Для доказательства второй части теоремы выражения $O\left(t^{-\beta-1} \ell(t)\right)$ и $O\left(t^{-4 / 3} \ell(t)\right)$ в (85) следует заменить на $\asymp t^{-\beta-1} \ell(t)$ и $\asymp t^{-4 / 3} \ell(t)$, если верно $(81)$, и на $\sim \beta D_{l m}^{*} t^{-\beta-1} \ell(t)$ и $\sim t^{-4 / 3} D_{l m}^{*} \ell(t) / 3$, если верно $(82)$.

Зафиксируем произвольное $\varepsilon \in(0,0.5)$. Тогда по лемме 5 с учетом свойств медленно меняющихся функций и соотношения (6) имеем

$$
\begin{aligned}
& \int_{\varepsilon t}^{(1-\varepsilon) t} P_{l 1}(t-u) P_{m 1}(t-u) \mathrm{d} G_{2}(u)=O\left(\mu_{2}^{-2}(t)\right)\left(1-G_{2}(t)\right) \\
&=\left\{\begin{array}{ccc}
o\left(t^{-\beta-1} \ell(t)\right), & \text { если } & \beta \in(0,1 / 2), \\
o\left(\mu_{2}^{-2}(t)\right), & \text { если } & \beta \in(0,1],
\end{array}\right. \\
& \int_{0}^{\varepsilon t} P_{l 1}(t-u) P_{m 1}(t-u) \mathrm{d} G_{2}(u) \sim P_{l 1}(t) P_{m 1}(t) \sim \frac{D_{l 1} D_{m 1}\left(\mu_{1} \beta \Gamma_{\beta}\right)^{2}}{\mu_{2}^{2}(t)} .
\end{aligned}
$$


Далее, при любом фиксированном $\nu \in(0,1)$ запишем представление

$$
\begin{aligned}
& S(t, \varepsilon):=\int_{(1-\varepsilon) t}^{t} P_{l 1}(t-u) P_{m 1}(t-u) \mathrm{d} G_{2}(u) \\
= & \left(\int_{(1-\varepsilon) t}^{t-t^{\nu}}+\int_{t-t^{\nu}}^{t}\right) P_{l 1}(t-u) P_{m 1}(t-u) \mathrm{d} G_{2}(u) \\
=: & S_{1 \nu}(t, \varepsilon)+S_{2 \nu}(t) .
\end{aligned}
$$

Если $\beta \in(0,1 / 2]$, то, используя $(7)$, получаем

$$
S(t, \varepsilon)=\int_{1}^{\varepsilon t} \frac{O(1) \ell(t) \mathrm{d} u}{t^{\beta+1} u^{2-2 \beta} \ell^{2}(u)}= \begin{cases}O\left(t^{-\beta-1} \ell(t)\right), & \text { если } \beta \in(0,1 / 2), \\ o\left(\mu_{2}^{-2}(t)\right), & \text { если } \beta \in(1 / 3,1 / 2] .\end{cases}
$$

Оценки (86)-(88) доказывают два первых равенства в (85), а также третье при $\beta \in(1 / 3,1 / 2]$, т.е. для всех $\beta \in(0,1 / 2]$. Это влечет (80) при $\beta \in(0,1 / 2]$. Если $\beta \in(0,1 / 3]$, то из условия (81) следует $S(t, \varepsilon) \asymp t^{-\beta-1} \ell(t)$, а из условия $(82)$

$$
S(t, \varepsilon) \sim \beta t^{-\beta-1} \ell(t) \int_{0}^{\infty} P_{l 1}(u) P_{m 1}(u) \mathrm{d} u=\beta t^{-\beta-1} \ell(t) D_{l m}^{*} .
$$

Данные оценки, соотношение (6) и цепочка равенств

$$
P_{l 1}(t) P_{m 1}(t)=O\left(\mu_{2}(t)\right)=O\left(t^{2-2 \beta}\right)=o\left(t^{-\beta-1}\right)
$$

при $\beta \in(0,1 / 3)$, а также асимптотические соотношения

$$
P_{l 1}(t) P_{m 1}(t) \sim \frac{D_{l 1} D_{m 1}\left(\left(\mu_{1} / 3\right) \Gamma_{1 / 3}\right)^{2}}{\mu_{2}^{2}(t)} \sim \frac{9 D_{l 1} D_{m 1}\left(\left(\mu_{1} / 3\right) \Gamma_{1 / 3}\right)^{2}}{4 t^{4 / 3} \ell^{2}(t)}
$$

при $\beta=1 / 3$ доказывают вторую часть леммы для $\beta \in(0,1 / 3]$.

Перейдем к случаю $\beta \in(1 / 2,2 / 3) \bigcup(2 / 3,1]$. В силу леммы 5 и условия $(5)$

$$
S_{1 \nu}(t, \varepsilon) \leqslant \varepsilon C_{3} \mu_{2}^{-2}\left(t^{\nu}\right) t^{-\beta} \ell(t)
$$

для некоторого $C_{3}>0$. После подстановки в правую часть явного выражения для $\mu_{2}(t)$ получаем, что она с точностью до медленно меняющейся функции имеет порядок $t^{(2 \beta-2) \nu-\beta}$.

Если $(2 \beta-2) \nu-\beta<2 \beta-2$, или $\nu>1-0.5 \beta /(1-\beta)$, то оценка (89) принимает вид

$$
S_{1 \nu}(t, \varepsilon)=o\left(\mu_{2}^{-2}(t)\right) .
$$

Мы рассматриваем только значения $\nu \in(0,1)$. Тогда соотношение (90) будет верно при всех $\nu \in(0,1)$, если $\beta \in(2 / 3,1]$. Если же $\beta \in(1 / 2,2 / 3)$, то подходящими будут только $\nu \in\left(1-0.5 \beta(1-\beta)^{-1}, 1\right)$.

При $\beta \in(2 / 3,1]$ по лемме 5 и условию (5)

$$
S_{2 \nu}(t, \varepsilon)=o\left(\mu_{2}^{-2}\left(t^{\nu}\right) t^{\nu-\beta} \ell(t)\right)
$$

где степенная часть справа имеет вид $t^{(2 \beta-1) \nu-\beta}$. Неравенство $t^{(2 \beta-1) \nu-\beta}<t^{2 \beta-2}$ перепишем в виде

$$
\nu<1-\frac{1-\beta}{2 \beta-1} \in(0,1], \text { если } \beta \in(2 / 3,1],
$$


и для таких $\nu>0$ оценка (91) преобразуется в

$$
S_{2 \nu}(t, \varepsilon)=o\left(\mu_{2}^{-2}(t)\right) .
$$

В итоге, при $\beta \in(2 / 3,1]$ существует такое $0<\nu<1-\frac{1-\beta}{2 \beta-1}$, что из (90) и (92) следует

$$
S(t, \varepsilon)=o\left(\mu_{2}^{-2}(t)\right),
$$

и, учитывая (86) и (87), мы доказали соответствующую часть утверждений (85) и (80).

Согласно лемме 5 и условию (78), если $\beta \in(1 / 2,2 / 3)$, то

$$
S_{2 \nu}(t, \varepsilon) \leqslant o\left(t^{(2 \beta-1) \nu+\phi(\beta)} \ell^{-2}\left(t^{\nu}\right)\right) .
$$

Докажем, что при $\beta \in(1 / 2,2 / 3)$ существуют такие $\nu \in\left(1-0.5 \beta(1-\beta)^{-1}, 1\right)$ и $\delta>0$, что $(2 \beta-1) \nu+\phi(\beta)-\delta<2 \beta-2$. Тогда, как и в случае $\beta \in(1 / 2,2 / 3)$, можно вывести оценку (93) и соответствующие части утверждений (85) и (80).

Если $\nu>1-0.5 \beta(1-\beta)^{-1}$, то $\phi(\beta)-\delta<2 \beta-2-(2 \beta-1)\left(1-0.5 \beta(1-\beta)^{-1}\right)$ при всех $\delta>0$. Заметим, что $\phi(\beta)=-\beta-3 / 2+(2-2 \beta)^{-1} \leqslant 2-2 \beta$, причем $\phi(1 / 2)=-3 / 2$ и $\phi(2 / 3)=-2 / 3$. С другой стороны, если $\phi(\beta)=-\beta-3 / 2+(2-2 \beta)^{-1}-\delta$ при некотором $\delta>0$, то

$$
\nu<1-\frac{\beta}{2(1-\beta)}+\frac{\delta}{2 \beta-1},
$$

и существует требуемое $\nu \in\left(1-0.5 \beta(1-\beta)^{-1}, 1\right)$ для $\delta>0$ достаточно малых. Это завершает доказательство соотношения (80) для всех случаев, кроме $\beta=2 / 3$.

В случае $\beta=2 / 3$ необходимо другое разбиение области интегрирования. А именHO,

$$
\begin{aligned}
S(t, \varepsilon) & =\left(\int_{(1-\varepsilon) t}^{t-\ell_{\#}^{-2}(t)}+\int_{t-\ell_{\#}^{-2}(t)}^{t}\right) P_{l 1}(t-u) P_{m 1}(t-u) \mathrm{d} G_{2}(u) \\
& =: \quad S_{1}(t, \varepsilon)+S_{2}(t) .
\end{aligned}
$$

Очевидно, что

$$
\begin{aligned}
& S_{1}(t, \varepsilon)=\left(\int_{(1-\varepsilon) t}^{t-\sqrt{t}}+\int_{t-\sqrt{t}}^{t-\ell_{\#}^{-2}(t)}\right) P_{l 1}(t-u) P_{m 1}(t-u) \mathrm{d} G_{2}(u) \\
\leqslant & C_{1} t^{-1 / 3} l^{2}(\sqrt{t}) \int_{t-\sqrt{t}}^{(1-\varepsilon) t} \mathrm{~d} G_{2}(u)+C_{2} \ell_{\#}^{4 / 3}(t) \ell^{2}\left(\ell_{\#}^{-2}(t)\right) \int_{t-\sqrt{t}}^{t-\ell_{\#}^{-2}(t)} \mathrm{d} G_{2}(u) \\
= & o\left(t^{-2 / 3} \ell(t) \ell_{\#}(t)\right)=o\left(t^{-2 / 3} \ell^{2}(t)\right) .
\end{aligned}
$$

При дополнительном условии (79) имеем

$$
S_{2}(t)=G_{2}\left(t+\ell_{\#}^{-2}(t)\right)-G_{2}(t)=o\left(\left(1-G_{2}(t)\right) \ell(t)\right)
$$

и

$$
S(t, \varepsilon)=o\left(t^{-2 / 3} \ell^{2}(t)\right)=o\left(\mu_{2}^{-2}(t)\right) .
$$

Отметим, что выполнение условия (79) при $\beta=2 / 3$ существенно только при $\ell(t)=o(1)$. Это завершает доказательство леммы 6 . 
В заключение заметим, что можно предложить и другие достаточные условия для справедливости условий (79). Например, $G_{2}(t+1)-G_{2}(t)=o\left(t^{-2 / 3-\rho} \ell(t)\right)$ при некотором $\rho>0$.

Отметим, что в утверждении леммы 18 из [1] в случае $\beta=1 / 3$ имеется неточность в определении постоянной, что в данной лемме 6 исправлено. Однако явный вид постоянной в этом техническом утверждении на основной результат леммы 18 из [1] - асимптотику функции $P_{i 1}^{(2)}(t)$ - не влияет.

Введем функцию $\ell_{0.5}(t):=\int_{1}^{t} \frac{\mathrm{d} u}{u \ell^{2}(u)}, \ell_{0.5}(t) \in \mathcal{L}$. Отметим, что $\ell^{-2}(t)=o\left(\ell_{0.5}(t)\right)$. Приведем обобщение леммы 19 из работы [1], отличающееся только условиями и тем, что в доказательстве вместо леммы 18 из [1] с ограничительным условием В нужно воспользоваться леммой 6 настоящей работы.

Теорема 7. Пусть верна Гипотеза $\boldsymbol{A}$ и выполнены условия (78) и (79). Тогда для $i=1,2$

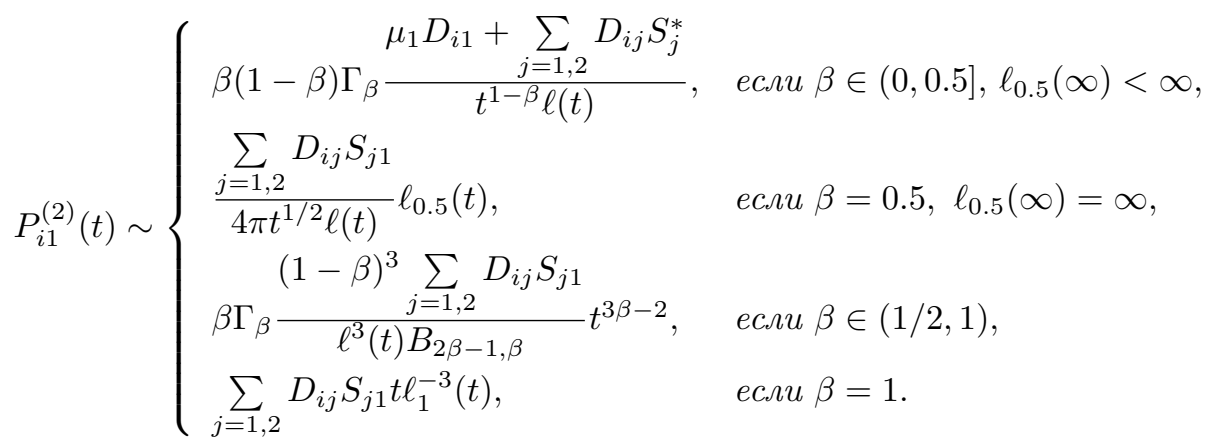

Автор выражает огромную благодарность рецензенту за кропотливое изучение работы и полезные рекомендации, что способствовало существенному улучшению текста статьи и устранению ряда пробелов в доказательств.

\section{Список литературы}

1. Ватутин В. А., Топчий В. А., "Критические ветвящиеся процессы Беллмана-Харриса с долго живущими частицами", Труды Матем. ин-та им. В. А. Стеклова, 282:2 (2013), $257-287$.

2. Ватутин В. А., “Дискретные предельные распределения числа частиц в ветвящихся процессах Беллмана-Харриса с несколькими типами частиц", Теория вероятн. и ее примен., 24:3 (1979), 503-514.

3. Erickson K. B., "Strong renewal theorems with infinite mean", Trans. Amer. Math. Soc., 151 (1970), 263-291.

4. Ватутин В. А., Топчий В. А., “Основная теорема восстановления для распределений с тяжелыми хвостами, имеющими индекс $\beta \in(0,0.5]$ ", Теория вероятн. и ее примен., $\mathbf{5 8 : 2}(2013), 387-396$.

5. Феллер В., Введение в теорию вероятностей и ее приложения, т. 2, пер. с англ., Мир, Москва, 1984, $751 \mathrm{c}$.

6. Bingham N. H., Goldie C. M., Teugels J. L., Regular variation, Cambridge Univ. Press, Cambridge, 1989, 494 c.

7. Topchii V., "Renewal measure density for distributions with regularly varying tails of order $\alpha \in(0,1 / 2]$ ", In: Workshop on Branching Processes and Their Applications, Lect. Notes Statist., 197, Springer, Berlin, 2010, 109-118. 
8. Топчий В. А., “Асимптотика производных от функции восстановления для распределений без первого момента с правильно меняющимися хвостами степени $\beta \in(0.5,1]$ ”, Дискретная математика, 24:2 (2012), 123-148.

9. Топчий В. А., "Производная плотности восстановления с бесконечным моментом при $\alpha \in(0,1 / 2]$ ]", Сиб. электр. матем. изв., 7 (2010), 340-349.

10. Сенета Е., Правильно меняющиеся функиии, пер. с англ., Наука, Москва, 1985, 141 с.

Статья поступила 17.12.2014. 